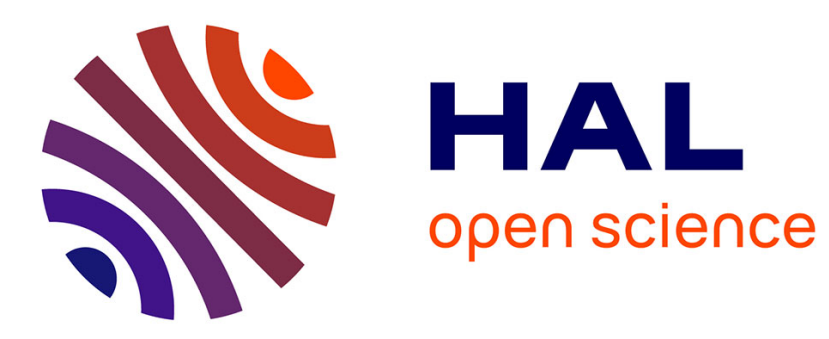

\title{
Elastic materials with couple-stresses
}

\author{
R. A. Toupin
}

\section{- To cite this version:}

R. A. Toupin. Elastic materials with couple-stresses. Archive for Rational Mechanics and Analysis, 1962, 11 (1), pp.385-414. hal-00852443

\section{HAL Id: hal-00852443 \\ https://hal.science/hal-00852443}

Submitted on 27 Aug 2013

HAL is a multi-disciplinary open access archive for the deposit and dissemination of scientific research documents, whether they are published or not. The documents may come from teaching and research institutions in France or abroad, or from public or private research centers.
L'archive ouverte pluridisciplinaire HAL, est destinée au dépôt et à la diffusion de documents scientifiques de niveau recherche, publiés ou non, émanant des établissements d'enseignement et de recherche français ou étrangers, des laboratoires publics ou privés. 


\title{
Elastic Materials with Couple-stresses
}

\author{
R. A. TOUPIN
}

Contents

1. Introduction . . . . . . . . . . . . . . 385

2. The equations of balance . . . . . . . . . . . . . . . 385

3. The dual representation of couple-stress . . . . . . . . . . . . 388

4. The irreducible parts of the couple-stress . . . . . . . . . . . . . . . . 390

5. The constitutive relations for a class of elastic materials with couple-stresses 394

6. An alternative representation for the stress and couple-stress . . . . . . 399

7. A principle of virtual work for the equations of static equilibrium . . . . 400

8. Equipollent distributions of stress and couple-stress . . . . . . . . . 405

9. Sound waves in elastic materials with couple-stresses . . . . . . . . . . 407

References . . . . . . . . . . . . . . . . 4 413

\section{Introduction}

Professor MINDLIN has brought to our attention an error in the equation for the couple-stress given in The Classical Field Theories. ${ }^{1}$ The results presented here grew from correspondence and discussions with MINDLIN on various aspects of the theory of perfectly elastic materials with couple-stresses which correspond to what was called in [CFT, §256A] the fully recoverable case. A thorough exposition of the linearized theory by MindiIn \& Tiersten appears as the following article in this Archive.

Our principal objective here is a representation for the energy, stress, and couple-stress, valid in the general non-linear case of finite strain and rotation. We wish also to review the basic concepts and foundations of the classical theory [2], to suggest a natural generalization of it, to discuss some qualitative features of wave propagation in elastic materials with couple-stresses, and to correct the equation in $[\mathrm{CFT}]$ for the couple-stress.

\section{The equations of balance}

Underlying the mechanics of continuous media are the principles of conservation or balance of mass, linear momentum, angular momentum, and energy.

A typical equation of balance has the general form

$$
\frac{d}{d t} \int_{v} \boldsymbol{Q} d v=\oint_{0} \boldsymbol{F} \cdot d \boldsymbol{a}+\int_{\nu} \boldsymbol{S} d v
$$

${ }^{1}$ Reference [1], Eq. (256.11) 2 . We shall refer to this article as [CFT] and follow its general scheme of notation and terminology where possible. 
where $r$ is a material region, $s$ is the complete boundary of $v, \boldsymbol{Q}$ is the density of the quantity in balance, $\boldsymbol{F}$ is its influx relative to the material (the efflux of a quantity is the negative of its influx), and $S$ is its source.

If a source vanishes, the corresponding quantity is conserved, and its equation of balance (2.1) reduces to an equation of conservation. If its influx also vanishes, the amount of the quantity assigned initially to a given set of material points is invariant in time. In classical mechanics, mass is such a quantity. Thus letting $\varrho$ denote the density of mass, we have the law of conservation of mass

$$
\frac{d}{d t} \int_{0} \varrho d v=\frac{d}{d t} \int_{0} d M=0
$$

Next we express the balance of (material) linear momentum, angular momentum, and energy by means of the equations ${ }^{1}$

$$
\begin{aligned}
& \frac{d}{d t} \int_{0} o_{0} \pi_{i} d M=\oint_{0} t_{i}^{j} d a_{j}+\int_{v} f_{i} d v, \\
& \frac{d}{d t} \int_{0} \sigma_{0} \sigma_{i j} d M=\oint_{0} \mu_{i}{ }^{k} d a_{k}+\int_{\nu} l_{i i} d v, \\
& \frac{d}{d t} \int_{0} E d M=\oint_{0} \mathrm{o} h^{k} d a_{k}+\int_{\nu} \mathrm{o} q d v .
\end{aligned}
$$

The influx of linear momentum, $\boldsymbol{t}$, is the stress tensor and its source $\boldsymbol{f}$ is the body force. These two quantities alone of the nine densities, influxes, and sources introduced in the three equations of balance (2.3) satisfy the principle of material indifference explained in [CFT, §293]. The remaining seven fields of (2.3) do not transform as tensors (i.e., are not invariant) under time dependent rigid transformations of the frame of reference. Moreover, as we know, the definition of angular momentum in a given frame of reference depends upon a reference point $\boldsymbol{O}$. For this reason we have appended a suffix $O$ to the left of the fields in (2.3) which depend upon the frame of reference.

The sources of linear momentum, angular momentum, and energy arise from interactions of the material with a gravitational or electromagnetic field. ${ }^{2}$

The density, influx and source of angular momentum are related to linear momentum, stress, and body force as follows:

$$
\begin{aligned}
\mathrm{o}^{\sigma_{i j}} & =2_{\mathrm{o}} x_{[i} \pi_{j]}+\sigma_{i j}=-\mathrm{o}_{\mathrm{o}} \sigma_{i i}, \\
\mathrm{o} \mu_{i, k} & =2_{\mathrm{O}} x_{[i} t_{j] k}+\mu_{i j k}=-{ }_{\mathrm{o}} \mu_{j i k}, \\
\mathrm{o}^{l_{i j}} & =2_{\mathrm{O}} x_{[i} f_{j]}+l_{i j}=-\mathrm{o}_{j i},
\end{aligned}
$$

${ }^{1}$ For the purpose of writing $(2.3)_{1}$ and $(2.3)_{2}$, we assume that the coordinate system is rectilinear so that the vector and tensor valued integrals which appear in these equations have an invariant significance.

${ }^{2}$ As pointed out on [CFT, \$225], it is possible to introduce gravitational and electromagnetic momentum and energy and thence to view the equations of balance (2.3) as equations of conservation of total (material plus field) momentum and energy. Within this view, body forces, and sources of angular momentum and energy represent rates as which the material gains (or loses) energy ani momentum from (or to) the gravitational or electromagnetic field. 
where we shall assume that the spin angular momentum per unit mass $\sigma$, the couple-stress $^{1} \boldsymbol{\mu}$, and the body couple $\boldsymbol{l}$ are independent of $\boldsymbol{O}$ and satisfy the principle of material indifference.

In (2.4), $\mathrm{o}^{x}$ denotes the position vector of a field point $\boldsymbol{x}$ relative to the reference point $\boldsymbol{O}$. According to (2.4), we may define spin angular momentum as the excess of angular momentum above the moment of linear momentum, couple-stress as the excess of influx of angular momentum above the moment of stress, and body couple as the excess of the source of angular momentum above the moment of body force. In the simpler theories of continuous media, each of these residuals vanishes. ${ }^{2}$

We take up next some relations between energy, momentum, and angular momentum. When the linear momentum per unit mass of a continuous medium is assumed equal to its velocity $\dot{x}$ and the spin angular momentum vanishes $(\sigma=0)$ as in the classical theories of elasticity and of ordinary fluids, the kinetic energy per unit mass is defined by

$$
\mathrm{o}^{x} \equiv \int^{t} \dot{\boldsymbol{x}} \cdot \dot{\boldsymbol{\pi}} d t+C,
$$

where $C$ is a constant of integration. Here, when the spin angular momentum is not necessarily zero, we generalize the definition (2.5) of kinetic energy as follows:

$$
\mathrm{o}^{x} \equiv \int^{t}(\dot{\boldsymbol{x}} \cdot \dot{\boldsymbol{\pi}}+\boldsymbol{\omega} \wedge \dot{\boldsymbol{\sigma}}) d t+C
$$

where $\omega_{i j}=x_{[i, i]}$ is the vorticity tensor and $\boldsymbol{\omega} \wedge \dot{\sigma}=\frac{1}{2} \omega_{i j} \dot{\sigma}^{i j}$ is the interior product (cf. \$3) of the vorticity and the rate of change of spin. Since $\dot{\boldsymbol{x}} \rightarrow \dot{\boldsymbol{x}}+\boldsymbol{u}$ under a change of frame, it is clear that, in general, kinetic energy is not invariant under all rigid transformations of the spatial coordinates. Alongside the relations (2.4) between the position vector of a field point, and linear and angular momentum, we now set the following relations between velocity, vorticity, linear and angular momentum, and energy:

$$
\begin{aligned}
\mathrm{o}^{E} & =\mathrm{o}^{\varkappa+\varepsilon} \\
\mathrm{o}^{h^{i}} & =\dot{x}^{j} t^{i}{ }_{j}+\frac{1}{2} \omega_{j k} \mu^{\prime k i}+h^{i}, \\
\mathrm{o} q & =\dot{x}^{i} f_{i}+\frac{1}{2} \omega_{i j} i^{i j}+q,
\end{aligned}
$$

where the internal energy $\varepsilon$, the extra flux of energy $\boldsymbol{h}$, and the extra source of energy $q$ are independent of $O$; i.e., these residuals are independent of the frame of reference. The first two terms in the energy flux $(2.7)_{2}$ are called the rate of working of the stress and the rate of working of the couple-stress, respectively. Similar terminology applies to the first two terms in oq.

1 The couple-stress $\boldsymbol{m}$ of [CFT] differs from the couple-stress $\mu$ as defined above by a factor of $2: m=\frac{1}{2} \mu, \mu=2 m$. The reason for making this slight change in terminology will become apparent in $\$ 3$ where we discuss the dual representation of angular momentum and couple-stress.

2 ERICKSEN, however, in a series of papers [3-6] has considered a theory of anisotropic fluids in which one or more of these residuals fails to vanish and the conservation laws must, for consistency of the whole scheme, be generalized along the lines just presented. 
Substituting the relations (2.4) and (2.7) into the integral equations of balance (2.2) and (2.3), we find that, in regions where all the fields are continuously differentiable, they are equivalent to the following system of field equations:

$$
\begin{aligned}
& \frac{\partial \varrho}{\partial t}+\left(\varrho \dot{x}^{i}\right)_{, i}=0, \\
& \varrho \ddot{x}_{i}=t_{i, j}^{j}+t_{i}, \\
& \varrho \dot{\sigma}_{i j}=2 t_{[j i]}+\mu_{i{ }^{k}, k}+l_{i j}, \\
& \varrho \dot{\varepsilon}=t^{(i j)} d_{i j}+\frac{1}{2} \mu^{i j k} \omega_{i j, k}+h^{k}{ }_{, k}+q,
\end{aligned}
$$

where $d_{i j}=\dot{x}_{(i, j)}=\frac{1}{2}\left(\dot{x}_{i, j}+\dot{x}_{j, i}\right)$ is the rate of deformation tensor. For ease of reference, $(2.8)_{2}$ is called the momentum equation; $(2.8)_{1}$, the continuity equation; $(2.8)_{3}$, the spin equation; and $(2.8)_{4}$, the energy equation.

\section{The dual representation of couple-stress}

In $\S 2$, angular momentum is represented by an antisymmetric tensor of rank t'wo. In 3-dimensional space, every such tensor has an alternative dual representation by an axial vector. For ease of comparison of our analysis of couple-stress with other treatments using the dual representation of angular momentum, we shall describe the relationship between the two schemes in this section.

Let $a_{i}$ and $b_{i}$ denote the covariant components of two arbitrary vectors $\boldsymbol{a}$ and $\boldsymbol{b}$. The exterior product ${ }^{1} \boldsymbol{a} \vee \boldsymbol{b}$ is an antisymmetric tensor of rank 2 which may be defined in terms of its components by

$$
(\boldsymbol{a} \vee \boldsymbol{b})_{i j} \equiv 2 a_{[i} b_{j]} .
$$

More generally, the triple exterior product of three vectors $\boldsymbol{a}, \boldsymbol{b}$, and $\boldsymbol{c}$ may be defined by

$$
(\boldsymbol{a} \vee \boldsymbol{b} \vee \boldsymbol{c})_{i j k} \equiv 3 ! a_{[\imath} b_{j} c_{k]} .
$$

Now let $e_{i j k}$ and $c^{i j k}$ denote the permutation symbols uniquely defined by the relations $e_{[i j k]}=e_{i j k}, e^{[i j k]}=C^{i j k}, e_{123}={ }^{[123}=1$. It is possible to view the permutation symbols as the components of two distinct relative tensors $\mathfrak{e}$ and Relative tensors are not true tensors since their definition always depends in some way upon the coordinate system or basis vectors. With each choice of coordinate system about a spatial point $\boldsymbol{x}$ or a material point $\boldsymbol{X}$, there corresponds a certain basis $\underset{p}{e}, p=1,2,3$ in the tangent space at $\boldsymbol{x}$, or in the tangent space at $\boldsymbol{X}$. The exterior product of the basis vectors $\underset{p}{e}$ taken in their natural order

$$
\boldsymbol{e} \equiv \underset{1}{e} \underset{2}{e} \underset{3}{e} \underset{3}{e}
$$

defines the relative tensor $\leftarrow$, and the exterior product of the reciprocal basis vectors $\stackrel{P}{\boldsymbol{e}}, p=1,2,3$ taken in their natural order defines

$$
\boldsymbol{E} \equiv \stackrel{1}{\boldsymbol{e}} \vee \stackrel{2}{\mathbf{e}} \vee \stackrel{3}{\mathbf{e}}
$$

1 For a concise, complete, and elegant exposition of the Grassman algebra and analysis of antisymmetric tensor fields (exterior differential forms) see WhITNEx [7]. 
A coordinate transformation induces a transformation of the basis $\underset{p}{e}$ according to the rule

$$
\underset{p}{\overline{\boldsymbol{e}}}=\frac{\partial x^{q}}{\partial \bar{x}^{p}} \underset{q}{\boldsymbol{e}},
$$

and one readily verifies that the relation between $\overline{\boldsymbol{e}}$ and $\boldsymbol{e}$ is

$$
\overline{\mathbf{e}}=(\overline{\boldsymbol{x}} / \boldsymbol{x}) \mathbf{e},
$$

where $(\bar{x} / x) \equiv \operatorname{det}\left\|\frac{\partial \bar{x}^{p}}{\partial x^{q}}\right\|$ is the Jacobian of the transformation $x \rightarrow \bar{x}$. In like manner we find that

$$
\overline{\mathbf{C}}=(\overline{\boldsymbol{x}} / \boldsymbol{x})^{-1} \mathbf{c} .
$$

Thus $\mathbf{e}$ and depend on the coordinate system and do not represent true tensors having a geometrical significance independent of the basis. Nevertheless, their components $e_{i j k}$ and $c^{i j k}$ enjoy the peculiar property that they. have the same values in every coordinate system; namely, $1,-1$, or 0 .

The dual of an antisymmetric tensor $a^{p q}=-a^{q p}$ is the relative vector $a$ defined by

$$
a_{r} \equiv \frac{1}{2} e_{p q r} a^{p q} .
$$

The components of $\mathfrak{a}$ determine the components of $a$ uniquely for

$$
a^{p q}=\mathfrak{a}_{r} \mathfrak{c}^{p q r} .
$$

Using the definition and notation $a \wedge b$ for the interior product of two antisymmetric tensors (ct. Whitney $[7, \S 7]$ ), we can write $(3.8)$ and $(3.9)$ in the form

$$
\begin{aligned}
& a=e \wedge a, \\
& a=a \wedge
\end{aligned}
$$

The dual of the exterior product of two vectors is the cross product:

$$
\operatorname{dual}(\boldsymbol{a} \wedge \boldsymbol{b})=e \wedge(\boldsymbol{a} \vee \boldsymbol{b}) \equiv \boldsymbol{a} \times \boldsymbol{b} .
$$

We shall also use the notations

$$
\begin{aligned}
& (\boldsymbol{n} \cdot \boldsymbol{a})^{i j k} \cdots=n_{p} a^{p i j k \ldots,}, \\
& (\boldsymbol{a} \cdot \boldsymbol{n})^{i j k \cdots}=a^{i j k \ldots p} n_{p},
\end{aligned}
$$

for the dot product of a vector and a tensor from the left and right.

In terms of these absolute notations, we can write (2.4) in the form

$$
\begin{aligned}
{ }_{0} \sigma & ={ }_{0} x \vee \pi+\sigma, \\
\mathrm{o}^{\mu} \cdot \boldsymbol{n} & ={ }_{\mathrm{o}} \boldsymbol{x} \vee(\boldsymbol{t} \cdot \boldsymbol{n})+\boldsymbol{\mu} \cdot \boldsymbol{n}, \\
{ }_{\mathrm{o}} & ={ }_{\mathrm{o}} \boldsymbol{x} \vee \boldsymbol{f}+\boldsymbol{l},
\end{aligned}
$$

where $(3.13)_{2}$ holds for an arbitrary vector $n$. Equivalently, taking the dual of these relations and using (3.11), we get

$$
\begin{aligned}
\operatorname{dual}_{\mathrm{o}} \boldsymbol{\sigma} & ={ }_{\mathrm{o}} \boldsymbol{x} \times \boldsymbol{\pi}+\hat{\boldsymbol{\sigma}}, \\
\operatorname{dual}\left({ }_{\mathrm{o}} \boldsymbol{\mu} \cdot \boldsymbol{n}\right) & ={ }_{\mathrm{o}} \boldsymbol{x} \times(\boldsymbol{t} \cdot \boldsymbol{n})+\hat{\boldsymbol{\mu}} \cdot \boldsymbol{n}, \\
\operatorname{dual}_{\mathrm{o}} \boldsymbol{l} & ={ }_{\mathrm{o}} \boldsymbol{x} \times \boldsymbol{f}+\hat{\boldsymbol{l}} .
\end{aligned}
$$


In these equations $\hat{\boldsymbol{\sigma}}$ and $\hat{\boldsymbol{l}}$ are the duals of the spin angular momentum $\boldsymbol{\sigma}$ and the body couple $l$ as defined in (3.8) and (3.9), but the dual couple-stress $\hat{\mu}$ is defined by

$$
\hat{\mu}_{i}^{k} \equiv \frac{1}{2} e_{i p q} \mu^{p q k}
$$

in terms of the couple-stress $\boldsymbol{\mu}$ and the relative tensor $\mathfrak{e}$. Note that $\hat{\boldsymbol{\mu}}$ is not the ordinary dual of $\mu$ which, if $\mu$ were an antisymmetric tensor, would be the axial scalar $\mathfrak{M}=\mathfrak{e} \wedge \mu=\frac{1}{3 !} e_{i j k} \mu^{i j k}$.

The dual couple-stress is a relative tensor, whose definition depends on the coordinate system. However, its components in any coordinate system uniquely determine the components of the couple-stress according to the formula

$$
\mu^{p q k}=\mathrm{e}^{p q r} \mu_{r}^{k} \text {. }
$$

\section{The irreducible parts of the couple-stress}

It is common and familiar procedure in continuum mechanics to decompose a covariant or contravariant tensor of rank two into a symmetric and an antisymmetric part. It is also common procedure to decompose a mixed tensor of rank two into a deviatoric part and a scalar part proportional to the unit tensor. If $a^{p q}$ are the components of an arbitrary contravariant tensor $\boldsymbol{a}$ of rank two, then

$$
\boldsymbol{a}={ }_{\mathrm{S}} \boldsymbol{a}+{ }_{\mathrm{A}} \boldsymbol{a},
$$

where the symmetric part $\mathrm{S}_{\mathrm{S}} \boldsymbol{a}$ and the antisymmetric part ${ }_{\mathrm{A}} \boldsymbol{a}$ have components given by

$$
\begin{aligned}
& { }_{\mathrm{s}} a^{p q}=\frac{1}{2}\left(a^{p q}+a^{q p}\right)=a^{(p q)}, \\
& { }_{\mathrm{A}} a^{p q}=\frac{1}{2}\left(a^{p q}-a^{q p}\right)=a^{[p q]} .
\end{aligned}
$$

We can write (4.1) and (4.2) in the form

$$
\begin{aligned}
\boldsymbol{a} & ={ }_{\mathrm{S}} \boldsymbol{I} \cdot \boldsymbol{a}+{ }_{\mathrm{A}} \boldsymbol{I} \cdot \boldsymbol{a}, \\
\mathrm{s} & ={ }_{\mathrm{S}} \boldsymbol{I} \cdot \boldsymbol{a}, \quad{ }_{\mathrm{A}} \boldsymbol{a}={ }_{\mathrm{A}} \boldsymbol{I} \cdot \boldsymbol{a},
\end{aligned}
$$

where ${ }_{S} \boldsymbol{I}$ and ${ }_{\mathrm{A}} \boldsymbol{I}$ are certain linear transformations in the vector space $V^{n^{2}}$ of dimension $n^{2}$ consisting in the set of all contravariant tensors of rank two. If one then considers the group of all tensor transformations in $V^{n^{2}}$, i.e., the set of all transformations having the form

$$
\bar{a}^{p q}=L^{p}{ }_{m}{ }^{q}{ }_{n} a^{m n},
$$

induced in $V^{n^{2}}$ by the group of all non-singular transformations $\{\boldsymbol{L}\}$ of the underlying vector space $V^{n}$, the subspaces $V^{\left(n^{2}\right)}$ and $V^{\left[n^{2}\right]}$ of $V^{n^{2}}$ consisting in all symmetric and all antisymmetric tensors of rank two, respectively, are invariant subspaces of $V^{n^{2}}$ under this group. Moreover, the linear operators ${ }_{S} I$ and ${ }_{A} I$ satisfy the equations

${ }_{\mathfrak{a}} \boldsymbol{I} \cdot{ }_{\mathfrak{b}} \boldsymbol{I}=0, \quad \mathfrak{a} \neq \mathfrak{b} ; \quad{ }_{\mathfrak{a}} \boldsymbol{I} \cdot{ }_{\mathfrak{b}} \boldsymbol{I}={ }_{\mathfrak{a}} \boldsymbol{I}, \quad \mathfrak{a}=\mathfrak{b} ; \quad \mathfrak{a}, \mathfrak{b}=\mathrm{S}, \mathrm{A}, \quad{ }_{\mathrm{S}} \boldsymbol{I}+{ }_{\mathrm{A}} \boldsymbol{I}=\boldsymbol{I}$,

where $I$ is the identity transformation of $V^{n^{2}}$. This decomposition of $V^{n^{2}}$ into a sum of two invariant subspaces is irreducible in the sense that, under the group of tensor transformations (4.4) induced in $V^{n^{2}}$ by the full linear group 
of transformations of $V^{n}$, there exists no finer decomposition of $V^{n^{2}}$ into invariant subspaces. This means that the only linear invariants of a second-order tensor under this group are its symmetry, antisymmetry, or asymmetry. There are of course non-linear invariants such as rank and signature.

The components of the symmetry operators ${ }_{S} I$ and ${ }_{A} I$ have the same values for every choice of basis and are given explicitly by

$$
\begin{aligned}
& { }_{\mathrm{s}} I_{r s}^{p q}=\frac{1}{2}\left(\delta_{r}^{p} \delta_{s}^{q}+\delta_{r}^{q} \delta_{s}^{p}\right)=\delta_{r}^{(p} \delta_{s}^{q)}, \\
& { }_{\mathrm{A}} I_{r s}^{p q}=\frac{1}{2}\left(\delta_{r}^{p} \delta_{s}^{q}-\delta_{r}^{q} \delta_{s}^{p}\right)=\delta_{r}^{[p} \delta_{s}^{q]}=\frac{1}{2} \delta_{r s}^{p q} .
\end{aligned}
$$

It is natural to ask if tensors of rank three and higher might also be written as sums of symmetry parts with properties similar to those just described for tensors of rank two. Indeed, WEYL 1 has developed a fully general theory of decomposition of tensors of arbitrary rank into irreducible symmetry parts which includes as a special case the foregoing familiar one for tensors of rank two. WEYL has demonstrated the relevance of this purely. algebraic theory to quantum mechanics. In fact, he apparently was led to pose the general mathematical question from a consideration of the quantum mechanical problem of decomposing the wave function of a system of identical particles into irreducible symmetry parts. But there has been little occasion to apply it in continuum mechanics except, of course, in the special case of tensors of rank two. The theory of materials considered here with its fundamental tensor of rank three, the couple-stress tensor, provides a non-trivial, and, as we shall see, useful application.

It turns out that tensors of rank three, which interest us at the moment, have four irreducible symmetry parts. There are four symmetry operators, which we denote by ${ }_{a} I, \mathfrak{a}=\mathrm{S}, \mathrm{A}, \mathbf{P}$, and $\overline{\mathrm{P}}$, replacing the two symmetry operators ${ }_{S} I$ and ${ }_{A} I$ of (4.3), (4.5), and (4.6). They satisfy the equations

and

$$
\begin{aligned}
& { }_{\mathfrak{a}} \boldsymbol{I} \cdot{ }_{\mathfrak{b}} \boldsymbol{I}={ }_{\mathfrak{a}} \boldsymbol{I} \text {, if } \mathfrak{a}=\mathfrak{b} \text {, } \\
& =0, \quad \text { if } \quad \mathfrak{a} \neq \mathfrak{b},
\end{aligned}
$$

$$
{ }_{\mathrm{S}} \boldsymbol{I}+{ }_{\mathrm{A}} \boldsymbol{I}+{ }_{\mathrm{P}} \boldsymbol{I}+{ }_{\mathrm{P}} \boldsymbol{I}=\boldsymbol{I} \text {. }
$$

Since we shall have considerable calculations to perform with , the symmetry parts of the couple-stress, we write out the components of the symmetry operators for this case explicitly. The first two are given by

$$
\begin{aligned}
& { }_{\mathrm{s}} I_{a b_{c}}^{p q_{r}}=\delta_{a}^{(p} \delta_{b}^{q} \delta_{c}^{p)}, \\
& { }_{\mathrm{A}} I_{a}^{p q_{c}}=\delta_{a}^{(p)} \delta_{b}^{q} \delta_{c}^{r]}=\frac{1}{3 !} \delta_{a b_{c}}^{p q}
\end{aligned}
$$

and might appropriately be called the symmetrizer and antisymmetrizer. The remaining two idempotents ${ }_{\mathrm{P}} \boldsymbol{I}$ and ${ }_{\mathrm{p}} \boldsymbol{I}$ are given explicitly by the formulas

$$
\begin{aligned}
& { }_{\mathrm{p}} I_{a b c}^{p q r} \div \frac{1}{3}\left(\delta_{a}^{p} \delta_{b}^{q} \delta_{c}^{r}+\delta_{a}^{\gamma} \delta_{b}^{q} \delta_{c}^{p}-\delta_{a}^{q} \delta_{b}^{p} \delta_{c}^{q}-\delta_{a}^{q} \delta_{b}^{p} \delta_{c}^{q}\right), \\
& \overline{\mathrm{p}} I_{a}^{p} q_{c}^{r}=\frac{1}{3}\left(\delta_{a}^{p} \delta_{b}^{q} \delta_{c}^{\gamma}+\delta_{a}^{q} \delta_{b}^{p} \delta_{c}^{r}-\delta_{a}^{z} \delta_{b}^{q} \delta_{c}^{p}-\delta_{a}^{q} \delta_{b}^{r} \delta_{c}^{p}\right) .
\end{aligned}
$$

${ }^{1}$ See $[8,9]$. Schouten [10] apparently initiated a general theory of such a decomposition. It is fully discussed by LiTTLEwood [11], and the paper by WADE [12] contains a bibliography of the subject. 
Let the corresponding parts of a general tensor $a$ of rank three be denoted by ${ }_{\mathrm{S}} \boldsymbol{a},{ }_{\mathrm{A}} \boldsymbol{a},{ }_{\mathrm{P}} \boldsymbol{a}$, and ${ }_{\mathrm{P}} \boldsymbol{a}$. We call $\mathrm{s}_{\mathrm{s}} \boldsymbol{a}$ the symmetric part, ${ }_{\mathrm{A}} \boldsymbol{a}$ the antisymmetric part, and ${ }_{\mathrm{p}} \boldsymbol{a}$ and ${ }_{\mathrm{p}} \boldsymbol{a}$ the parts with mixed symmetry.

The generalization of (4.2) to tensors of rank three is thus

$$
\begin{aligned}
& { }_{\mathrm{s}}^{a^{p q r}}=\frac{1}{6}\left(a^{p q r}+a^{q r p}+a^{r p q}+a^{p q q}+a^{r q p}+a^{q p r}\right), \\
& { }_{\mathrm{A}} a^{p g r}=\frac{1}{6}\left(a^{p q r}+a^{q r p}+a^{r q p}-a^{p q q}-a^{r q p}-a^{q p r}\right) ; \\
& { }_{\mathrm{p}} a^{p q r}=\frac{1}{3}\left(a^{p q r}+a^{r q p}-a^{q p r}-a^{r p q}\right), \\
& \overline{\mathrm{p}} a^{p q r}=\frac{1}{3}\left(a^{p q r}+a^{q p r}-a^{r q p}-a^{q p r}\right) .
\end{aligned}
$$

We now apply these general considerations of tensors of rank three to the couple-stress tensor $\mu^{p q r}$. The couple-stress tensor, by definition, is antisymmetric in its first pair of indices:

$$
\mu^{p q r}=-\mu^{q p r} .
$$

Thus it is not a general tensor of rank three. Its irreducible symmetry parts can be found by substituting $\mu$ in the relations (4.11) and simplifying the results using (4.12). In this way we find that

$$
\begin{aligned}
& \mathrm{s} \mu^{p q r}=0, \\
& { }_{\overline{\mathrm{P}}} \mu^{p q r}=0, \\
& \mu_{\mathrm{A}} \mu^{p q r}=\frac{1}{3}\left(\mu^{p q r}+\mu^{r p q}+\mu^{q r p}\right), \\
& \mu_{\mathrm{P}} \mu^{p q r}=\frac{1}{3}\left(2 \mu^{p q r}+\mu^{r q p}-\mu^{r p q}\right) .
\end{aligned}
$$

We call ${ }_{\mathrm{P}} \mu$ the principal part of the couple-stress. It follows from (4.13) that only the principal part and the antisymmetric part of the couple-stress fail to vanish. There are eight independent components of the principal part and only one independent component of the antisymmetric part. The principal part is determined uniquely by the part $\mu^{p(q r)}$ and conversely, for

$$
\begin{aligned}
\mathrm{P}^{p} \mu^{p r} & =\frac{2}{3}\left(\mu^{p(q r)}-\mu^{q(p r)}\right), \\
\mu^{p(q r)} & =\frac{1}{2}\left({ }_{\mathrm{P}} \mu^{p q r}+{ }_{\mathrm{P}} \mu^{p r q}\right) \\
& ={ }_{\mathrm{P}} \mu^{p(q r)} .
\end{aligned}
$$

We insert a caution with regard to the notation ${ }_{\mathrm{p}} \mu^{p(q)}$. This symbol stands for the quantity on the right-hand side of $(4.14)_{2}$ and not for the principal part of the tensor $\mu^{p(q r)}$. For the latter quantity it is necessary to use some other notation such as ${ }_{\mathrm{P}}\left[\mu^{p(q)}\right]$. The tensor $\mu^{p(q r)}$ is not an irreducible symmetry part of the couple-stress. We have, rather,

$$
\begin{aligned}
& \mathrm{s}\left[\mu^{p(q r)}\right]=0, \quad \mathrm{~A}\left[\mu^{p(q r)}\right]=\mathrm{O}, \\
& \mathrm{P}\left[\mu^{p(q r)}\right]=\frac{1}{6}\left(2 \mu^{p q r}+\mu^{p r q}-\mu^{q r p}\right), \\
& \overline{\mathrm{P}}\left[\mu^{p(q r)}\right]=\frac{1}{6}\left(2 \mu^{p r q}+\mu^{p q r}-\mu^{\gamma q p}\right) .
\end{aligned}
$$

It follows from (4.13) and (4.15) that

$$
\begin{aligned}
\mu^{p q r} & ={ }_{\mathrm{p}} \mu^{p q r}+{ }_{\mathrm{A}} \mu^{p q r} \\
\mu^{p(q r)} & ={ }_{\mathrm{P}}\left[\mu^{p(q r)}\right]+\overline{\mathrm{P}}\left[\mu^{p(q r)}\right],
\end{aligned}
$$


and from (4.14) and (4.16) 1 that the tensors $\mu^{p(q r)}$ and ${ }_{A} \mu^{p q r}$ determine the couple-stress uniquely. Setting $\nu^{p g r} \equiv \mu^{p(q r)}$, we have

$$
\mu^{p q r}=\frac{4}{3} \nu^{[p q] r}+{ }_{A} \mu^{p q r} \text {. }
$$

We now consider the corresponding invariant decomposition of the dual couple-stress $\hat{\mu}_{p}^{q}$ which is given in terms of the couple-stress by (3.14). Mixed tensors, or relative tensors, of rank two may be decomposed into a traceless or deviatoric part and a scalar part proportional to the unit tensor, and this decomposition corresponds to an irreducible, invariant decomposition of the space of mixed tensors of rank two under the group of mixed tensor transformations $\overline{\boldsymbol{a}}=\boldsymbol{L} \cdot \boldsymbol{a} \cdot \boldsymbol{L}^{-1}$, where $\boldsymbol{L}$ runs over the full linear group. For the dual couple-stress, this decomposition is as follows:

$$
\begin{aligned}
\hat{\boldsymbol{\mu}} & ={ }_{D} \hat{\boldsymbol{\mu}}+\frac{1}{3} \operatorname{tr} \hat{\boldsymbol{\mu}} \boldsymbol{I} \\
\operatorname{tr}_{D} \hat{\boldsymbol{\mu}} & =0 .
\end{aligned}
$$

In terms of components, these equations read

$$
\begin{aligned}
\hat{\mu}_{p}{ }^{4} & ={ }_{\mathrm{D}} \hat{\mu}_{p}{ }^{q}+\frac{1}{3} \hat{\mu}_{k}{ }^{k} \delta_{\dot{p}}{ }^{q} . \\
\mathrm{D} \hat{\mu}_{k}{ }^{k} & =\mathrm{O} .
\end{aligned}
$$

We observe next that the antisymmetric part ${ }_{A} \mu$ of the couple-stress has only one independent component and that we may always write it in the form

$$
{ }_{\mathrm{A}} \mu^{p q \gamma}=\mathfrak{M} \mathbb{E}^{p q \psi},
$$

where the relative scalar $\mathfrak{M}$ is given by

$$
\mathfrak{R}=\frac{1}{3 !} e_{p q r} \mu^{p q r}
$$

Calculation now reveals that the dual, $\hat{A}_{\mathrm{\mu}_{s}}{ }^{r}=\frac{1}{2} \mathrm{e}_{s p q \mathrm{~A}} \mu^{p q r}$, of the antisymmetric part of the couple-stress is equal to the scalar part of the dual couple-stress; i.e.,

$$
{ }_{\mathrm{A}} \hat{\mu}=\frac{1}{3} \operatorname{tr} \hat{\mu} I,
$$

and the dual of the principal part of the couple-stress, ${ }_{\mathrm{p}} \hat{\mu}_{s}^{\gamma}=\mathrm{e}_{p q r} \mathrm{p} \mu^{p q \gamma}$, is equal to the deviatoric part of the dual couple-stress:

$$
{ }_{\mathrm{P}} \hat{\boldsymbol{\mu}}={ }_{\mathrm{D}} \hat{\boldsymbol{\mu}} \text {. }
$$

Thus the dual of equation (4.16) ${ }_{1}$ which decomposes the couple-stress into irreducible symmetry parts is precisely equation (4.18), which decomposes the dual conple-stress into its deviatoric and scalar parts.

Equations $(4.15)_{1}$ and $(4.15)_{2}$ have important consequences in $\S 5$. There we shall make use of the trivial

Lemma 4.1. Necessary and sufficient conditions for a tensor equation

$$
A^{p q \ldots}=B^{p q \ldots}
$$

to be satisfied are that each of the tensor equations

$$
{ }_{\mathrm{a}} A^{p q \ldots}={ }_{\mathfrak{a}} B^{p q \ldots}, \quad \mathfrak{a}=1,2, \ldots, N
$$


be satisfied, where ${ }_{a} \boldsymbol{A}$ and ${ }_{\mathrm{a}} \boldsymbol{B}$ are the irreducible symmetry parts of $\boldsymbol{A}$ and $\boldsymbol{B}$. Sufficiency follows on noting that $\sum_{a}{ }_{a} \boldsymbol{A}=\boldsymbol{A}$ and $\sum_{\mathfrak{a}} \boldsymbol{B}=\boldsymbol{B}$; necessity follows from the linearity of the symmetry operators.

\section{The constitutive relations for a class of elastic materials with couple-stresses}

This section defines a class of elastic materials by a sequence of constitutive assumptions (CA 1), (CA 2),... . We introduce these special assumptions one at a time with explanations for the less transparent ones.

CA 1. These elastic materials shall have a natural state $C_{0}$. The natural state is a relaxed configuration of the material points in which the stress, couplestress, and extra flux of energy all vanish. The temperature $\theta$ of the natural state is uniform, and the material is at rest in an inertial frame of reference. We shall identify the material coordinates $X^{\alpha}(\boldsymbol{X})$ of a material point $\boldsymbol{X}$ as the coordinates of its position in the relaxed configuration $C_{0}$. By the motion of the material we shall mean the smooth mappings $x^{i}=\hat{x}^{i}(X, t)$ giving the position $\boldsymbol{x}$ of each material point $\boldsymbol{X}$ for each instant of time $t$.

CA 2. As in classical elasticity theory, we shall assume that the momentum per unit mass is equal to the velocity $\left.\dot{x}^{i} \equiv \frac{\partial \hat{x}^{i}}{\partial t}\right|_{\boldsymbol{X}}$

CA 3. The internal energy is a function of: a) the nire components $x_{; \alpha}^{p} \equiv \frac{\partial \hat{x}^{p}}{\partial X^{a}}$ of the deformation gradient of the present configuration $C(t)$ relative to the relaxed configuration $C_{0}$; b) the eighteen independent components $x_{; \alpha \beta}^{p}$ of the material gradient of the deformation gradient; and c) the entropy density $\eta$. Thus we have

$$
\varepsilon=\hat{\varepsilon}\left(x_{; \alpha}^{p}, x_{; \alpha \beta}^{p}, \eta\right) .
$$

Rather than choosing 18 of the 27 variables $x_{; \alpha \beta}^{p}$ arbitrarily as independent variables in the energy function, we regard $c$ as a function of all 27 but subject to the nine constraints

$$
\hat{\varepsilon}\left(x_{; \alpha}^{p}, x_{; \alpha \beta}^{p}, \eta\right)=\hat{\varepsilon}\left(x_{; \alpha}^{p}, x_{; \beta \alpha}^{p}, \eta\right)
$$

so that, in particular, we have

for all values of $p, \alpha$, and $\beta$.

$$
\frac{\partial \bar{\varepsilon}}{\partial x_{; \alpha \beta}^{p}}=\frac{\partial \bar{\varepsilon}}{\partial x_{; \beta \alpha}^{p}}
$$

CA 4. The materials are fully recoverable in the sense explained in [CFT, $\S 256 \mathrm{~A}]$, and the extra flux of energy $\boldsymbol{h}$ is heat flux. This means, in particular, that we assume

$$
\varrho \theta \dot{\eta}=h^{p}, p+q,
$$

as in classical elasticity theory. ${ }^{1}$ We call Eq. (5.4), the heat equation.

${ }^{1}$ In $\S \S 7$ and 8 we give arguments which indicate that the hypotheses of CA 4 concerning the extra flux of energy $h$ are too sestrictive. A more general and natural assumption splits the extra flux of energy into a sum $h^{p}=H^{p q^{\gamma}} d_{q r}+q^{p}$ of heat flux $\boldsymbol{q}$ and a rate of working of the boundary by deformation. We shall see that, within this view, setting $H^{p q r}=0$ imposes an unnatural restriction upon the stored elastic energy function $\bar{\varepsilon}$. 
It follows from CA 3 and CA 4 that the energy equation (2.3), can be put in the form ${ }^{1}$

$$
\begin{aligned}
\left(\varrho \frac{\partial \hat{\varepsilon}}{\partial x_{; \alpha}^{p}}\right. & \left.-g_{p r} t^{(r q)} X_{; q}^{\alpha}-\frac{1}{2} \mu_{p}^{q r} X_{; q r}^{\alpha}\right) \dot{x}_{; \alpha}^{p}+ \\
& +\left(\varrho \frac{\partial \hat{\varepsilon}}{\partial x_{; \alpha \beta}^{p}}+\frac{1}{2} \mu_{p}^{q r} X_{; q}^{\alpha} X_{;}^{\beta}\right) \dot{x}_{; \alpha \beta}^{p}+ \\
& +\varrho\left(\frac{\partial \hat{\varepsilon}}{\partial \eta}-\theta\right) \dot{\eta}=0 .
\end{aligned}
$$

Since $X_{; q r}^{\alpha}=X_{; \gamma q}^{\alpha}$, and $x_{; \alpha \beta}^{p}=x_{; \beta \alpha}^{p}$, we see that the couple-stress $\mu_{p}^{q r}$ may be replaced throughout the energy equation (5.5) by its part $\nu_{p}^{q r}=\mu_{p}^{(q r)}$. According to (4.4) the part $\mu_{p}^{(g r)}$ of the couple-stress is independent of the antisymmetric part of the couple-stress (scalar part of the dual couple-stress); hence, the internal energy equation (5.5) can yield no relation between the energy of the material and the antisymmetric part of the couple-stress. Equation (5.5) is also independent of the antisymmetric part of the stress and depends only on the symmetric part $t^{(p q)}$. These facts, the structure of the energy equation (5.5) and the heat equation (5.4), and experience with simpler theories of elasticity lead us to

CA 5. The stress, couple-stress, and temperature in the materials considered here are functions of the same variables which suffice to determine the value of the internal energy; moreover, these constitutive relations are such as to satisfy the internal energy equation (5.5) identically. In other words, equation (5.5) is not to restrict the motion or entropy fields in any way.

The conditions of CA 5 are met if and only if the constitutive relations for the symmetric part of the stress, the part $\mu^{p(q)}$ of the couple-stress, and the temperature are related to the energy function by the following formulas: ${ }^{2}$

$$
\begin{aligned}
t^{(p q)} & =\varrho\left[\frac{\partial \hat{\varepsilon}}{\partial x_{p ; \alpha}} x_{i \alpha}^{q}+\frac{\partial \hat{\varepsilon}}{\partial x_{p ; \alpha \beta}} x_{; \alpha \beta}^{q}\right], \\
\mu^{p(q \gamma)} & =-2 \varrho \frac{\partial \hat{\varepsilon}}{\partial x_{p ; \alpha \beta}} x_{; \alpha}^{q} x^{\gamma}{ }_{; \beta}, \\
\theta & =\frac{\partial \hat{\varepsilon}}{\partial \eta} .
\end{aligned}
$$

We now apply Lemma (4.1) to the tensor equation (5.6) to obtain the two tensor equations equivalent to it:

$$
\begin{aligned}
& t^{(p q)}=\varrho\left[\frac{\partial \hat{\varepsilon}}{\partial x_{(p ; \alpha}} x_{; j \alpha}^{q)}+\frac{\partial \hat{\varepsilon}}{\partial x_{(p ; \alpha \beta}} x_{; \alpha \beta}^{q)}\right] \\
& \frac{\partial \hat{\varepsilon}}{\partial x_{[p ; \alpha}} x_{; \alpha}^{q]}+\frac{\partial \hat{\varepsilon}}{\partial x_{[p ; \alpha \beta}} x_{; \alpha \beta}^{q]}=0 .
\end{aligned}
$$

${ }^{1} C f .[C F T, \S 256]$ for a guide to the manipulations necessary to achieve (5.5).

2 The point raised by Mindin and mentioned in the Introduction is that, in Eq. (256.11) $)_{2}$ of [CFT], which in the fully recoverable case should reduce to (5.7), $m_{p}^{q}$; stands on the left rather than $m_{p}^{(q)}$. Setting the dissipative part of the couplestress equal to zero in $(256.11)_{2}$, we should then conclude that $m^{p[q+]}=0$ since the righthand side is symmetric in $q$ and $r$. But $m^{p[q r]}=0$ implies that $m^{p q r}=0$. This can be seen most easily by adding the three equations $m^{p q r}-m^{p: q}=0, m^{q r p}-m^{q} f r=0$, $m^{r q p}-m^{r p q}=0$, and using the fact that $m^{p q r}=-m^{q p r}$. It was not our intention that the couple-stress need vanish in the fully recoverable case. To correct $(256.11)_{3}$ as it appears in [CFT], place round brackets about the indices $q r$ on the left-hand side of it. 
Equation $(5.9)_{2}$ is a necessary and sufficient condition that the energy be invariant under all rigid motions (see e.g., $[13, \S 10]$ ). Thus we have

Theorem 5.1. The internal energy of an elastic material is invariant under all rigid motions.

This is a familiar result in the classical theory of elasticity, where invariance of the energy under rigid motions is necessary and sufficient for the symmetry of the stress tensor.

Next apply Lemma (4.1) to the tensor equation (5.7) to replace it by the following system of equivalent tensor equations:

$$
\begin{aligned}
\mathrm{P}\left[\mu^{p(q \gamma)}\right] & =-2 \varrho_{\mathrm{P}}\left[\frac{\partial \hat{\varepsilon}}{\partial x_{p ; \alpha \beta}} x_{; \alpha}^{q} x_{; \beta}^{\gamma}\right], \\
\overline{\mathrm{P}}\left[\mu^{p(q \gamma)}\right] & =-2 \varrho_{\overline{\mathrm{P}}}\left[\frac{\partial \hat{\varepsilon}}{\partial x_{p ; \alpha \beta}} x_{; \alpha}^{q} x_{; \beta}^{\gamma}\right], \\
\frac{\partial \hat{\varepsilon}}{\partial x_{(p, \alpha \beta}} x_{; \alpha}^{q} x_{; \beta}^{*)} & =0, \quad \frac{\partial \hat{\varepsilon}}{\partial x_{[p ; \alpha \beta}} x_{; \alpha}^{q} x_{; \beta}^{\gamma]}=0 .
\end{aligned}
$$

The single independent equation of $(5.10)_{4}$ is satisfied by every function $\hat{\varepsilon}$ that depends symmetrically upon $x_{; \alpha \beta}^{p}$ and $x_{; \beta \alpha}^{p}$, so that (5.3) holds. However, not every energy function satisfies the 10 independent equations $(5.10)_{3}$ even if it be rotationally invariant, so that $(5.9)_{2}$ holds.

Collecting results, we see that the energy function cannot depend arbitrarily upon the deformation gradient $x_{; \alpha}^{p}$ and its material gradient $x_{; \alpha \beta}^{p}$ but must satisfy the system of equations

$$
\begin{aligned}
\frac{\partial \hat{\varepsilon}}{\partial x_{p ; \alpha \beta}}-\frac{\partial \hat{\varepsilon}}{\partial x_{p ; \beta \alpha}} & =0, \quad \frac{\partial \hat{\varepsilon}}{\partial x_{[p ; \alpha}} x_{; \alpha}^{q]}+\frac{\partial \hat{\varepsilon}}{\partial x_{[p ; \alpha \beta}} x_{; \alpha \beta}^{q]}=0, \\
\frac{\partial \hat{\varepsilon}}{\partial x_{(p ; \alpha \beta}} x_{; \alpha}^{q} x_{; \beta}^{r)} & =0 .
\end{aligned}
$$

There are nine independent equations $(5.11)_{1}$, three independent equations $(5.11)_{2}$, and ten independent equations $(5.11)_{3}$. Thus the system of equations (5.11) comprises a total of $9+3+10=22$ first order homogeneous partial differential equations for the energy function $\hat{\varepsilon}$, and there is a total of $9+27+1=37$ independent variables $x_{; \alpha}^{p}, \quad x_{; \alpha \beta}^{p}$, and $\eta$. The general solution for $\hat{\varepsilon}$ is an arbitrary function of any $37-22=15$ functionally independent solutions of this system. Thirty-seven solutions of the system (5.11) are

$$
\eta, C_{\alpha \beta}=x_{p ; \alpha} x_{; \beta}^{p}, \quad D_{\alpha \beta \lambda}={ }_{P} C_{\alpha \beta, \lambda}=\frac{2}{3} C_{\lambda[\alpha, \beta]} .
$$

But they are not all functionally independent, since

$$
C_{[\alpha \beta]}=0, \quad D_{[\alpha \beta \lambda]}=0, \quad D_{(\alpha \beta \lambda)}=0, \quad \overline{\mathrm{p}} D_{\alpha \beta \lambda}=0 .
$$

Counting independent relations in (5.13), we find a total of $3+1+10+8=22$. Except for these relations amongst the set (5.12), they are functionally independent. Hence, amongst the quantities (5.12) one can always find 15 functionally independent solutions of the system (5.11) and we have the representation 
Theorem 5.2. The energy function of an elastic material as defined by the constitutive assumptions CA 1 to CA 5 is expressible as a function of the variables (5.12).

This theorem generalizes the known result of ordinary elasticity theory wherein the energy is always expressible as a function of the components $C_{\alpha \beta}$ of Green's deformation tensor $\boldsymbol{C}$. There it is always possible to express the energy as a function of any 6 independent components of $\boldsymbol{C}$, say $C_{11}, C_{22}, C_{33}$, $C_{12}, C_{23}$, and $C_{13}$. It is customary and more convenient for theoretical purposes, however, to regard the energy as a function of all nine components of the symmetric tensor $\boldsymbol{C}$ and to restrict the functional dependence of the energy in such a way that

$$
\tilde{\varepsilon}\left(C_{\alpha \beta}\right)=\tilde{\varepsilon}\left(C_{\beta \alpha}\right) .
$$

With this agreement we then have

or

$$
\frac{\partial \hat{\varepsilon}}{\partial C_{\alpha \beta}}=\frac{\partial \hat{\varepsilon}}{\partial C_{\beta \alpha}},
$$

$$
{ }_{A}\left(\frac{\partial \hat{\varepsilon}}{\partial C_{\alpha \beta}}\right)=0, \quad \text { and } \frac{\partial \hat{\varepsilon}}{\partial C_{\alpha \beta}}={ }_{\mathrm{S}}\left(\frac{\partial \hat{\varepsilon}}{\partial C_{\alpha \beta}}\right) .
$$

In the more general case now at hand we can follow a similar procedure. Rather than expressing the energy as a function of 6 independent components of $\boldsymbol{C}$ and 8 independent components of $\boldsymbol{D}$, we express it as a function $\tilde{\boldsymbol{\varepsilon}}$ of all 36 variables $C_{\alpha \beta}$ and $D_{\lambda \alpha \beta}$ (plus the entropy) and require that

$$
\begin{aligned}
{ }_{\mathrm{A}}\left(\frac{\partial \tilde{\varepsilon}}{\partial C_{\alpha \beta}}\right)=0, \quad{ }_{\mathrm{A}}\left(\frac{\partial \tilde{\varepsilon}}{\partial D_{\lambda \alpha \beta}}\right) & =0, \quad \mathrm{~S}\left(\frac{\partial \tilde{\varepsilon}}{\partial D_{\lambda \alpha \beta}}\right)=0, \\
\overline{\mathrm{P}}\left(\frac{\partial \tilde{\varepsilon}}{\partial D_{\lambda \alpha \beta}}\right) & =0 .
\end{aligned}
$$

We then have as a consequence of (5.17)

$$
\begin{gathered}
\frac{\partial \tilde{\varepsilon}}{\partial C_{\alpha \beta}}={ }_{\mathrm{S}}\left(\frac{\partial \tilde{\varepsilon}}{\partial C_{\alpha \beta}}\right), \\
\frac{\partial \tilde{\varepsilon}}{\partial D_{\lambda \alpha \beta}}={ }_{\mathrm{P}}\left(\frac{\partial \tilde{\varepsilon}}{\partial D_{\lambda \alpha \beta}}\right) .
\end{gathered}
$$

Making use of this agreement as to the functional form of the energy, we find that the expressions (5.6) and (5.7) for the stress and couple-stress reduce to the form

$$
\begin{aligned}
& t^{(p q)}=\varrho\left[2 \frac{\partial \tilde{\varepsilon}}{\partial C_{\alpha \beta}^{-\beta}} x_{; \alpha}^{p} x_{; \beta}^{q}+{ }_{3}^{4} \frac{\partial \tilde{\varepsilon}}{\partial D_{\lambda \times \beta}^{-}} x_{; \alpha}^{(p} x_{; \beta \lambda}^{q)}\right], \\
& \mu^{p(q)}=-\frac{4}{3} \varrho \frac{\partial \widetilde{\varepsilon}}{\partial D_{\lambda \alpha \beta}} x_{; \alpha}^{p} x_{\beta ;}^{(q} x_{; \lambda}^{\tau)} .
\end{aligned}
$$

CA 6. Under CA 5 we have shown how the constitutive relations for the symmetric part of the stress and the principal part of the couple-stress are determined by the energy function and the hypotheses made thus far. According to (4.17), this leaves the antisymmetric part of the couple-stress (equivalently, the scalar part of the dual couple-stress) undetermined by the energy 
function. In fact, we have seen that this part of the couple-stress has no energetic significance whatsoever. Constitutive relations for the antisymmetric part of the stress may also be assigned independently of the energy under the assumptions made thus far. Now the body-couples, like the body force and the energy source, are regarded here as assigned functions of $\boldsymbol{x}$ and $t$, so that, if constitutive relations for the antisymmetric parts of the stress and couple-stress were given, one could merely calculate the time rate of change $\dot{\sigma}_{i j}$ of spin angular momentum from $(2.8)_{3}$ once the motion and entropy field were known. But here we shall consider the special theory of elastic media for which it is postulated that the spin angular momentum is at most a constant along each material point. Thus we assume that

$$
\dot{\sigma}_{i j}=0
$$

But the equations of balance of linear momentum $(2.8)_{1}$ and the heat equation (5.4) when supplemented by constitutive relations for the stress, heat flux, and temperature comprise a set of 4 equations for determining the 4 unknowns $\hat{x}^{p}(\boldsymbol{X}, t)$ and $\eta(\boldsymbol{X}, t)$. In view of this and the hypothesis (5.20), which requires

$$
2 t_{[i j]}=\mu_{i j}^{k}{ }_{k}+l_{i j}
$$

we shall regard (5.21) as an identity. We may take it as a definition of the antisymmetric part of the stress in this special theory of elastic media with couplestresses. $^{1}$ Under this agreement, the only constitutive relations left to specify or determine are those for the heat flux and the antisymmetric part of the couple-stress (scalar part of the dual couple-stress). In other words, a material of the class considered here is completely characterized by giving its energy function, heat flux, and antisymmetric part of couple-stress as functions of the deformation $\hat{\boldsymbol{x}}(\boldsymbol{X}, t)$, entropy field $\eta(\boldsymbol{X}, t)$, and derivatives of these quantities.

With the antisymmetric part of the stress given by (5.21) we can eliminate it from the equation of balance of linear momentum $(2.8)_{2}$ to get the equations of motion

$$
\varrho \ddot{x}^{i}=t^{(i j)}{ }_{j}+\mu^{i j k}{ }_{, k j}+f^{i}+l^{i j}, j .
$$

But the double divergence $\mu^{i j k}{ }_{, j k}$ of the couple-stress which appears in the equations of motion is independent of the antisymmetric part of the couplestress, and (5.22) can be written in the form

$$
\varrho \ddot{x}^{i}=t^{(i j)}{ }_{, j}+\mu^{i(j k)}{ }_{, j k}+f^{i}+l^{i j}{ }_{j}
$$

1 More general treatments of spin angular momentum are conceivable but will not be discussed here. By introducing a more elaborate kinematical apparatus such as that considered by the CosSERATs [2], it is possible to consider gyroscopic elastic media for which $\dot{\sigma}_{i j} \neq 0$. The present work, however, restricts attention to a simple type of medium for which the motion $\hat{\boldsymbol{x}}(\boldsymbol{X}, t)$ and the entropy field $\eta(\boldsymbol{X}, t)$. suffice to determine all the relevant mechanical and thermal properties of the material. In this respect, the class of media considered here is the same as that considered in classical elasticity theory. The only generalization attempted here is to allow the energy, stress, and couple-stress to depend upon the gradient of the deformation gradient. Our analysis shows that if the energy depends on these higher derivatives of the displacement, then, in general, the principal part of the couple-stress cannot vanish in such a material. 
from which it is obvious that the energy function alone suffices to determine the form of the equations of motion provided that the body force and couple are regarded as prescribed functions of position and time. The constitutive relations for the heat flux are needed only for the heat equation (5.4), and the constitutive relation for the scalar part of the dual couple-stress is needed at most for the expression of certain types of boundary conditions.

\section{An alternative representation for the stress and couple-stress}

The eight independent components of the material tensor

$$
\begin{aligned}
D_{\alpha \beta \lambda} & ={ }_{\mathrm{P}} C_{\alpha \beta, \lambda}=\frac{2}{3} C_{\lambda[\beta, \alpha]} \\
& =-D_{\beta \alpha \lambda}
\end{aligned}
$$

may be represented in dual form by a relative material tensor of rank two defined by

where

$$
\begin{aligned}
\mathbb{D}_{\lambda}^{\zeta} & =\frac{1}{2} \mathbb{C}^{\zeta \zeta \beta} D_{\alpha \beta \lambda}, \\
D_{\alpha \beta \lambda} & =\mathfrak{e}_{\alpha \beta \zeta} \mathfrak{D}_{\lambda}^{\zeta},
\end{aligned}
$$

$$
\mathfrak{D}_{\zeta}^{\zeta} \equiv 0 \text {. }
$$

The nine components of $D$ are functionally independent except for the single relation (6.3). They are given explicitly in terms of $x_{p ; \alpha}$ and $x_{p ; \alpha \beta}$ by the formulas

$$
\begin{aligned}
\mathfrak{D}_{\lambda}^{\xi} & =\frac{1}{3} \mathbb{E}^{5 \alpha \beta} C_{\lambda \beta, \alpha} \\
& =\frac{1}{3} \mathbb{C}^{5 \alpha \beta}\left(x_{p ; \lambda} x_{; \alpha \beta}^{p}+x_{p ; \lambda \alpha} x_{; \beta}^{p}\right) \\
& =\frac{1}{3} \operatorname{cts}^{5 \alpha \beta} x_{p ; \lambda \alpha} x_{; \beta}^{p} .
\end{aligned}
$$

Also, for some purposes, it is convenient to replace Green's deformation tensor in the energy function by the strain tensor (cf. [CFT, \$31]):

$$
E_{\alpha \beta} \equiv \frac{1}{2}\left(C_{\alpha \beta}-g_{\alpha \beta}\right),
$$

where the $g_{\alpha \beta}$ are the components of the metric tensor in the material coordinate system. When the material is undeformed and in its natural state, $\boldsymbol{E}=0$. This property of the strain tensor makes it convenient for expansions of the energy function about the undeformed state. Since the set of variables $\mathfrak{D}_{\lambda}^{\zeta}$ and $E_{\alpha \beta}$ determine the set $D_{\alpha \beta \lambda}$ and $C_{\alpha \beta}$ uniquely, it is always possible to express the energy in the form

$$
\varepsilon=\bar{\varepsilon}\left(E_{\alpha \beta}, D_{\alpha}^{\zeta}, \eta\right) .
$$

But not all the components of $D$ and $\boldsymbol{E}$ are independent, so we restrict the functional dependence of the energy upon these variables in such a way that

$$
\delta_{\lambda}{ }_{\lambda} \frac{\partial \bar{\varepsilon}}{\partial \mathcal{D}_{\lambda}^{\zeta}}=0, \quad{ }_{\mathrm{A}}\left(\frac{\partial \bar{\varepsilon}}{\partial E_{\alpha \beta}}\right)=0 .
$$

The stress and couple-stress are then given by the formulas:

$$
\begin{aligned}
& t^{(p q)}=\varrho\left[\frac{\partial \bar{\varepsilon}}{\partial E_{\alpha \beta}} x_{; \alpha}^{p} x_{; \beta}^{q}+\frac{2}{3} \frac{\partial \bar{\varepsilon}}{\partial \mathcal{D}^{\zeta}}{ }_{\lambda}^{\left(\varepsilon^{5} \alpha \beta\right.} x_{; \beta \lambda}^{(p)} x_{; \alpha}^{q)}\right] .
\end{aligned}
$$

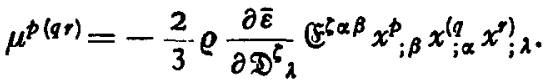


Multiplying (6.9) by $\mathrm{e}_{p q}$, and summing on $p$ and $q$, we get

$$
\mathrm{D} \hat{\mu}_{s}^{r}=\frac{2}{3} \varrho(\boldsymbol{x} / \boldsymbol{X}) \frac{\partial \bar{\varepsilon}}{\partial \mathcal{D}_{\lambda}^{\xi}}\left(X_{; s}^{\zeta} x_{; \lambda}^{r}-\delta_{s}^{r} \delta_{\lambda}^{\zeta}\right) \text {. }
$$

Since we assume (6.7), this simplifies to

where

$$
\mathrm{D}_{\dot{\mu}}^{\hat{\mu}_{s}^{\prime}}=\frac{2}{3} \varrho(\boldsymbol{x} / \boldsymbol{X}) \frac{\partial \bar{\varepsilon}}{\partial D^{\zeta} \lambda} x_{; \lambda}^{r} X_{; s}^{\zeta}
$$

$$
(\boldsymbol{x} / \boldsymbol{X}) \equiv \operatorname{det}\left\|x_{; \alpha}^{p}\right\| \text {. }
$$

\section{A principle of virtual work for the equations of static equilibrium}

The equations governing static equilibrium of elastic materials as these have been defined in the previous sections can be obtained as a special case of the foregoing dynamical analysis simply by setting all time rates equal to zero. This section derives and discusses these same equations by an independent analysis based upon a principle of virtual work. This treatment emphasizes energetics and the calculus of variations. The method begins with the concept of the stored elastic energy of a deformed elastic body $\mathscr{B}$. For definiteness, the body is here assumed to fill a regular region $\mathscr{V}$ of space in its undeformed, relaxed state. The boundary of $\mathscr{V}$ consists in the union of a finite number of smooth surfaces, smooth curves (edges), and points (corners). We consider only ' 3 -times differentiable deformations $\hat{\boldsymbol{x}}(\boldsymbol{X})$ giving the deformed configuration of the material points. The stored elastic energy is given by an integral

$$
\mathscr{E}=\int U d V
$$

where $U$ is the energy of the deformed state per unit undeformed volume. By introducing the energy per unit mass $\varepsilon=U / \varrho$, the stored energy may also be expressed as an integral

$$
\mathscr{E}=\int_{0} \varrho \varepsilon d v
$$

over the region $v$ occupied by the deformed equilibrium configuration of the material points. Since the mapping $\boldsymbol{X} \rightarrow \boldsymbol{x}$ is smooth, $v$ is also regular, and its boundary is the union $s$ of a finite number of smooth surfaces, plus the union $c$ of a finite number of smooth curyes (deformed edges), plus a finite number of isolated points (deformed positions of corners). The energy per unit undeformed volume, hence also the energy per unit mass in the deformed state, is assumed to be a function

$$
U=\hat{U}\left(x_{; \alpha}^{p}, x_{; \alpha \beta}^{p}, \boldsymbol{X}\right)
$$

of the deformation gradient, its material gradient, and, in inhomogeneous materials, the energy density may also depend on the material points $\boldsymbol{X}$. For simplicity, we neglect all thermal phenomena and do not consider the more general case where $U$ depends on the entropy density or temperature.

We focus attention upon a particular deformed equilibrium state of the material under the influence of a certain set of generalized forces. These generalized 
forces comprise volume, surface, and line distributions of force. We arrived at a suitable definition of these forces by the following line of reasoning. First, one introduces the concept of virtual displacements $\delta x_{p}$ of the equilibrium configuration of the material points. These are defined in the usual way by introducing a set or sets $\boldsymbol{x}=\widehat{\boldsymbol{x}}(\boldsymbol{X}, \lambda)$ of comparison states, where $\widehat{\boldsymbol{x}}(\boldsymbol{X}, 0)$ is the equilibrium configuration and $\left.\delta x^{p} \equiv \frac{\partial \hat{x}^{p}}{\partial \lambda}\right|_{\lambda=0}$. The first variation $\delta \mathscr{E}=d \mathscr{E}|d \lambda|_{\lambda=0}$ of the stored energy (7.1) or (7.2) is then expressible in the form

$$
\delta \mathscr{E}=\int_{\sigma} A^{i} \delta x_{i} d v+\oint_{\delta} B^{i} \delta x_{i} d a+\oint_{\delta} C^{i} D \delta x_{i} d a+\int_{c} D^{i} \delta x_{i} d l
$$

where $d v, d a$, and $d l$ are scalar elements of volume, area, and length, respectively, and $D \delta x_{i}$ denotes the normal derivative $e^{1}$ of the variation $\delta x_{i}$ at a boundary point of the body which is not an edge or corner.

Now it can be shown that an integral expression of the form (7.4) vanishes for arbitrary functions $\delta x_{i}$ if and only if $A^{i}=B^{2}=C^{i}=D^{i}=0$. In other words, the variations in (7.4) are, in this sense, independent. For each of these independent variations we introduce a generalized force $\boldsymbol{F}$, and postulate as a necessary condition of equilibrium that

$$
\delta \mathscr{E}=\delta \mathscr{W}=\int_{v} F^{i} \delta x_{i} d v+\oint_{0} F^{i} \delta x_{i} d a+\oint_{0} F^{i} D \delta x_{2} d a+\int_{c} F_{4} F^{i} \delta x_{\imath} d l,
$$

for arbitrary virtual displacements $\delta \boldsymbol{x}$.

1 In reducing $\delta \mathscr{E}$ to the form (7.5) we have also used the notion of the surface gradient $D_{k} \varphi$ of a function defined on the boundary of the body. For functions defined in the interior and on the boundary, the gradient, normal derivative, and surface gradient are related by

$$
\varphi, k=D \varphi n_{k}+D_{k} \varphi
$$

where $n_{k}$ is the unit normal to the surface. The second fundamental form $\boldsymbol{b}$ of a smooth part of the boundary is defined by

$$
b_{i j} \equiv-D_{i} n_{j}=-D_{j} n_{i}
$$

and we shall make repeated use of the integral identity

$$
\int_{\mathscr{S}} D_{i} f \ldots n_{j} d a=\int_{\mathscr{S}}\left(b^{k}{ }_{k} n_{i} n_{j}-b_{i j}\right) f d a+\oint_{\mathscr{C}} m_{i} n_{j} f \ldots d l
$$

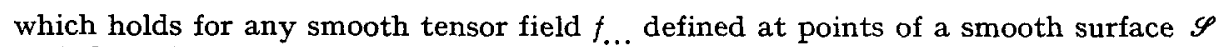
with boundary curve $\mathscr{C}$. The vector $\boldsymbol{m}=\boldsymbol{\tau} \times \boldsymbol{n}$, where $\boldsymbol{\tau}$ is the unit tangent to $\mathscr{C}$. If the integral transformation (A) be applied to each member of the finite collection of smooth surfaces on the boundary of a regular region, and the result summed, one gets the identity

$$
\int_{\sigma} D_{i} f \ldots n_{j} d a=\oint_{\sigma}\left(b_{k}^{k} n_{i} n_{j}-b_{i j}\right) f \ldots d a+\int_{c}\left[m_{\imath} n_{j} f_{\ldots}\right] d l
$$

where the bold-face brackets [ ] in the integral over the edges $c$ of the boundary denote the difference in values of the enclosed quantity as a given point on an edge is approached from either side. If the boundary of the region has no edges and $f \ldots$ is smooth throughout 5 , the line integral in (B) vanishes. 
If we set

$$
\begin{aligned}
\alpha^{p q} & =\varrho\left(\frac{\partial \hat{\varepsilon}}{\partial x_{p ; \alpha}} x_{; \alpha}^{q}+\frac{\partial \hat{\varepsilon}}{\partial x_{p ; \alpha \beta}} x_{; \alpha \beta}^{q}\right), \\
\beta^{p q r} & =\varrho \frac{\partial \hat{\varepsilon}}{\partial x_{p ; \alpha \beta}} x_{; \alpha}^{q} x_{; \beta}^{\prime}=\beta^{p r q},
\end{aligned}
$$

it is a matter of straightforward application of the divergence theorem, integration by parts, and the integral transformation (B) to reduce $\delta \mathscr{E}$ to the form

$$
\begin{aligned}
\delta \mathscr{E}= & \int_{v}\left(-\alpha^{p q}{ }_{, q}+\beta^{p q r}{ }_{, q r}\right) \delta x_{p} d v+ \\
& +\oint_{\sigma}\left\{\alpha^{p q} n_{p}-D \beta^{p q r} n_{q} n_{r}-2 D_{a} \beta^{p(q r)} n_{r}+\right. \\
& \left.+\left(b^{k}{ }_{k} n_{q} n_{r}-b_{q r}\right) \beta^{p q r}\right\} \delta x_{p} d a+ \\
& +\oint_{\sigma} \beta^{p q r} n_{q} n_{r} D \delta x_{p} d a+\int_{c}\left[m_{q} n_{r} \beta^{p q r}\right] \delta x_{p} d l .
\end{aligned}
$$

From (7.5) and (7.7) we deduce that necessary and sufficient conditions for the variation in energy to equal the virtual work in every virtual displacement from equilibrium are

$$
\begin{gathered}
-\alpha^{p q}{ }_{q}+\beta^{p q r}{ }_{q r}={ }_{1} F^{p}, \quad x \in v ; \\
\alpha^{p q} n_{q}-D \beta^{p q r} n_{q} n_{r}-2 D_{q} \beta^{p(q r)} n_{r}+\left(b_{k}^{k} n_{q} n_{r}-b_{q r}\right) \beta^{p q r}={ }_{2} F^{p}, \quad x \in \delta ; \\
\beta^{p q r} n_{q} n_{r}={ }_{3} F^{p}, \quad x \in s ; \\
{\left[m_{q} n_{r} \beta^{p q r}\right]={ }_{4} F^{p}, \quad x \in c .}
\end{gathered}
$$

These are the equations of equilibrium and boundary conditions corresponding to the principle of virtual work (7.5).

Now a rigid virtual displacement has the general form

$$
\delta x_{p}=a_{p}+e_{p q} \Re^{q} x^{\prime},
$$

in rectangular Cartesian coordinates, where $a_{p}$ and $\Re^{q}$ are arbitrary constants. If the total energy is invariant under rigid motions, by writing the variation of the energy in the form,

$$
\delta \mathscr{E}=\int_{\sigma}\left(\alpha^{p q} \delta x_{p, q}+\beta^{p q r} \delta x_{p, q r}\right) d v,
$$

we see that a necessary and sufficient condition for this invariance is

$$
\int_{-}^{[p q]} d v=0
$$

If the energy of every part of the body is separately invariant under all rigid variations, we must have

$$
\alpha^{[p q]}=0 \text {. }
$$

Now invariance of the energy under rigid motions imposes certain compatibility conditions upon the generalized forces ${ }_{1} F,{ }_{2} F,{ }_{2} F$, and $F$. From (7.5) 
we see that if $\delta \mathscr{E}=0$ for all rigid variations, then equilibrium is impossible unless

$$
\int_{1} F d v+\oint_{2} F d a+\int_{c} F d l=0,
$$

which follows from invariance of the energy under virtual translations, and

$$
\int x \times{ }_{1} F d v+\oint_{0} x \times{ }_{2} F d a+\oint_{0} \times{ }_{3} F d a+\int_{c} x \times{ }_{4} F d l=0,
$$

which follows from the invariance of the energy under virtual rotations. From these conditions of compatibility on the generalized forces, we can read off, at least in part, their mechanical significance: ${ }_{1} F$ is a volume distribution of force, ${ }_{2} F$ is a surface distribution of force, ${ }_{4} F$ is a line distribution of force over the edges of the body, and, the tangential component $n \times{ }_{3} \boldsymbol{F}$ of ${ }_{3} \boldsymbol{F}$ is a surface distribution of couples. The conditions of compatibility are independent of the normal component $n \cdot{ }_{3} F$ of the third generalized force; hence, this part of ${ }_{3} F$ has only energetic significance. We shall see in a moment that, in the theory of elastic equilibrium characterized by the constitutive assumptions CA 1 to CA 5 of $\$ 5$, the normal component of the third generalized force is assumed to be zero. To prove this, we first note that the energy equation $(2.3)_{3}$ with ${ }^{h} h^{i}$ given by (2.7) and with $h^{i}$ set equal to zero as the condition for neglect of thermal effects implies that the virtual work done on any portion of an elastic body in equilibrium is expressible in the special form

$$
\begin{aligned}
\delta \mathscr{W}= & \int f^{i} \delta x_{i} d v-\frac{1}{2} \int l^{i j} \delta x_{i, j} d v+ \\
& +\oint_{\sigma} t^{i j} \delta x_{i} n_{j} d a-\frac{1}{2} \int_{\sigma} \mu^{i j k} \delta x_{i, j} n_{k} d a .
\end{aligned}
$$

Integration by parts and application of the integral transformation (B) reduces this expression to the form

$$
\begin{aligned}
\delta \mathscr{W}= & \int\left(f^{i}+\frac{1}{2} l^{i j}{ }_{j}\right) \delta x_{i} d v+ \\
& +\oint^{i j} i^{i j} n_{j}-\frac{1}{2} \mu^{i j k}{ }_{, k} n_{j}-\frac{1}{2} l^{i j} n_{j}-\frac{1}{2}\left(b_{m}^{m} n_{j} n_{k}-b_{j k}\right) \mu^{i(j k)}+ \\
& \left.+D \mu^{i(j k)} n_{j} n_{k}+D_{k} \mu^{i(j k)} n_{j}\right\} \delta x_{i} d a+ \\
& -\frac{1}{2} \oint \mu^{i(j k)} n_{j} n_{k} D \delta x_{i} d a+\oint_{e}\left[m_{j} n_{k} \mu^{i j k}\right] \delta x_{i} d l
\end{aligned}
$$

in which the variations are now all independent. From this expression for the virtual work in terms of the stress, couple-stress, body force and couples, we see that the generalized forces have the special form

$$
\begin{aligned}
{ }_{1} F^{i}= & f^{i}+\frac{1}{2} l^{i j}{ }_{, j}, \\
{ }_{2} F^{i}= & t^{i i} n_{j}-\frac{1}{2} \mu^{i j k}{ }_{, k} n_{j}-\frac{1}{2} l^{i j} n_{j}+ \\
& -\frac{1}{2}\left(b_{m}^{m} n_{j} n_{k}-b_{j k}\right) \mu^{i(j k)} \frac{1}{2} D \mu^{i(j k)} n_{j} n_{k}+D_{k} \mu^{i(j k)} n_{j}, \\
{ }_{3} F^{i}= & -\frac{1}{2} \mu^{i(j k)} n_{j} n_{k}, \\
{ }_{4} F^{i}= & {\left[m_{j} n_{k} \mu^{i j k}\right] . }
\end{aligned}
$$


But the couple-stress is antisymmetric in its first two indices; hence, it follows immediately from $(7.19)_{3}$ that the generalized force ${ }_{3} F^{i}$ corresponding to the expression (7.17) for the virtual work has a vanishing normal component:

$$
{ }_{3} F^{i} n_{\imath}=-\frac{1}{2} \mu^{2(1) k} n_{\imath} n_{j} n_{k}=0 .
$$

Thus, in this special case,

$$
{ }_{3} \boldsymbol{F}=-\boldsymbol{n} \times\left(\boldsymbol{n} \times{ }_{3} \boldsymbol{F}\right)
$$

and ${ }_{3} \boldsymbol{F}$ is determined wholly by the part $\boldsymbol{n} \times{ }_{3} \boldsymbol{F}$, which, we have shown, corresponds to a surface distribution of couples.

From the expressions (7.19) for the generalized forces in terms of the stress and couple-stress and the boundary conditions (7.9) and (7.10), we may recover the basic relations (5.6) and (5.7) between energy, stress, and couple-stress, if it is assumed that the former must hold for every regular part of a body in equilibrium. From (7.10) and $(7.19)_{3}$ we first obtain the relation

$$
\mu^{i(j k)}=-2 \beta^{i j k},
$$

which is (5.7) in the present notation. Substituting this in the relation obtained by equating the right-hand side of $(7.19)_{2}$ and the left-hand side of (7.9), we get the equality

$$
t^{2 j}-\frac{1}{2} \mu^{2 j k}{ }_{, k}-\frac{1}{2} l^{2 j}=x^{2} .
$$

But, in equilibrium, the left-hand side of (7.23) is the symmetric part of the stress (ct. (2.8)), and (7.23) reduces to (5.6).

From the general energetic approach outlined in this section, the restriction

$$
{ }_{3} F^{2} n_{b}=0
$$

seems unnatural. From the considerations just given we may trace its origin to the constitutive assumption CA 4. The total flux of energy has the general form

$$
\mathrm{o}^{h^{i}}=\dot{x}^{i} t_{j}^{i}+\frac{1}{2} \omega^{j k} \mu_{j k}^{i}+h^{i},
$$

and, in CA 4, the extra flux of energy $h^{i}$ was identified as the heat flux. This assumption excludes the possibility of work terms having the form $H^{i j k} d_{j k}$ with $H^{i j k}=H^{i k j}$ arising from a local deformation of the boundary of the body. Relaxing the tacit assumption $\boldsymbol{H}=0$ of the classical theory brings it into precise correspondence with the more general theory of equilibrium of this section in which the generalized force ${ }_{3} \boldsymbol{F}$ may have a non-vanishing normal component. In this more general theory, the energy function need not satisfy the 10 independent conditions

$$
\beta^{(2 j k)}=0 \text {. }
$$

The energy function will be invariant under rigid motions if and only if $\alpha^{[\imath]}=0$. It can then be shown that every such energy function is expressible as a function of the $6+18$ independent variables $C_{\alpha \beta}$ and $C_{\alpha \beta, \lambda}$, which replace the $6+8$ independent variables $C_{\alpha \beta}$ and $C_{\alpha[\beta, \lambda]}$ of the restricted theory in which the 
energy must satisfy (7.26) as well as the condition of invariance under rigid motions. For convenience, we shall call (7.26), the Cosserat relations. In $\$ 9$ we shall obtain an interesting consequence of these relations which provides a possible test of their validity.

\section{Equipollent distributions of stress and couple-stress}

In the theory of elasticity characterized by the assumptions CA 1 to $\mathrm{CA} 5$ of $\$ 5$, the energy function determines the symmetric part of the stress-strain and the principal part of the couple-stress-strain relations. The assumption $\dot{\sigma}=0$ converts the equation of balance of angular momentum into a definition of the antisymmetric part of the stress in terms of the divergence of the couple-stress field. Thus to determine the state of stress and couple-stress completely in these materials it suffices to know the distribution of internal energy and the antisymmetric part of the couple-stress. At the end of $\S 5$ we pointed out that the equations of motions as well as the energy equation are independent of the antisymmetric part of the couple-stress. It is remarkable also that this part of the couple-stress does not appear in the boundary conditions of the variational principle of $\S 7$. The following discussion of equipollent distributions of stress and couple-stress is inserted in an attempt to understand the elusive nature of the antisymmetric part of the couple-stress.

Call any pair of distributions $(\boldsymbol{t}, \boldsymbol{\mu})$ and $\left(\boldsymbol{t}^{*}, \boldsymbol{\mu}^{*}\right)$ of stress and couple-stress equipollent if they yield the same values for the resultant force and moment on every part of a material body $\mathscr{B}$. Call any pair of such distributions of stress and couple-stress energetically equivalent if the symmetric part of the stress and the principal part of the couple-stress coincide at each point of the medium. Let square brackets about $[t]$ and $[\boldsymbol{\mu}]$ or any parts of these tensors denote the difference between a pair of distributions of stress and couple-stress. Thus a pair is equipollent if and only if

$$
\begin{array}{r}
\oint\left[t^{i j}\right] d a_{j}=0, \\
\oint\left(2_{0} x_{[i}\left[t_{j}{ }^{k}\right]+\left[\mu_{i j}{ }^{k}\right]\right) d a_{k}=0,
\end{array}
$$

for every closed surface with is the complete boundary of some part of the body. A pair of distributions will be equipollent and energetically equivalent if the symmetric part $\left[t^{(i j)}\right]=0$, the principal part $\left[\mathrm{p} \mu^{i j k}\right]=0$, and

$$
\begin{aligned}
\oint\left[{ }_{\mathrm{A}} t^{i j}\right] d a_{j} & =0, \\
\oint\left(2_{\circ} x^{[i}\left[{ }_{\mathrm{A}}{ }^{i] k}\right]+[\mathfrak{M}] \mathfrak{F}^{i j k}\right) d a_{k} & =0
\end{aligned}
$$

where we have set $\left[\mu^{[i j k]}\right]=[\mathfrak{M}] \mathbb{F}^{i j k}$. Now it is known ${ }^{1}$ that if $\boldsymbol{F}$ is any vector field in a star-shaped region such that

$$
\oint F^{i} d a_{i}=0
$$

for every closed surface in the region, then there exists an antisymmetric field $H^{i j}$ such that

$$
F^{i}=H^{i j}, \quad H^{i j}=-H^{j i}
$$


Equations (8.2) are three equations of the form (8.3); hence, there exists at least one tensor field of rank three, say $P^{i j k}$, such that

and we must have

$$
\left[t^{[i j]}\right]=P^{i j k}, \quad P^{i j k}=-P^{i k j},
$$

$$
P^{(i j) k}{ }_{, k}=0 .
$$

It follows from (8.6) that, in $(8.5)_{1}$, we may take $P^{i j k}$ to be completely antisymmetric. But, in three dimensions, this means that $P^{i j k}$ is expressible in the form $P^{i j k}=\mathfrak{P} \mathfrak{G}^{i j k}$ where $\mathfrak{P}$ is a relative scalar. Thus

$$
\left[t^{[i j]}\right]=\mathbb{C}^{i j k} \mathfrak{P}, k .
$$

Substituting this result in (8.2) ${ }_{2}$ and applying (8.4) again, we find that we must have

$$
2 \mathfrak{P}, r-[\mathfrak{M}]_{, r}=0,
$$

which proves the following theorem on equipollence:

Theorem 8.1. An energetically equivalent pair of distributions of stress and couple-stress are equipollent if and only if there exists a scalar $\mathfrak{B}$ such that the antisymmetric parts of the stress distributions are related by

$$
t^{[i j]}=t^{*[i]]}+\mathfrak{C}^{i j k} \mathfrak{B}, k,
$$

and the scalar parts of the couple-stress distributions are related by

$$
\mathfrak{M} \cdot \stackrel{\sim}{*}+2(\mathfrak{P}+\mathfrak{\Re}),
$$

where $\mathfrak{H}$ is an arbitrary constant.

Suppose one lays down the additional restriction upon a pair of equipollent and energetically equivalent distributions of stress and couple-stress that, at each point of the boundary of a given elastic body, the stress vector and couplestress vector coincide. That is, let us require that

$$
\begin{aligned}
{\left[t^{[i j]}\right] n_{j} } & =0, \\
{\left[\mu^{[i j k]}\right] n_{k} } & =0
\end{aligned}
$$

1 There is a striking resemblance between this group of transformations relating energetically equivalent and equipollent distributions of stress and couple stress and the gauge transformations of electromagnetic theory. The similarity is made more pronounced by introducing the vector invariant $\times \mathrm{t}_{i} \approx \frac{1}{2} \mathrm{e}_{i j k} t^{j k}$ of stress and writing (8.9) in the form

alongside,

$$
\begin{aligned}
& \times \mathfrak{t}={ }_{x} \mathbf{t}^{*}+\operatorname{grad} \mathfrak{P} \\
& \mathfrak{M}=\mathfrak{M}^{*}+2(\mathfrak{B}+\mathfrak{R}) .
\end{aligned}
$$

The gauge transformations on the electromagnetic scalar potential $V$ and vector potential $\boldsymbol{A}$ have the form

$$
\begin{aligned}
& \boldsymbol{A}=\boldsymbol{A}^{*}+\operatorname{grad} P, \\
& V=V^{*}-\partial P / \partial t,
\end{aligned}
$$

where $P$ is again an arbitrary scalar field. Hence the indeterminacy in the stress and couple-stress of equipollent, energetically equivalent distributions is analogous to the indeterminacy in the electromagnetic potential corresponding to a given electromagnetic field. 
at every point of a closed surface. This requires, in addition to (8.9) and (8.10), that

and

$$
\mathbb{C}^{i j k} n_{j} \mathfrak{B}_{, k}=0 \text {, }
$$

$$
[\mathfrak{M}] \mathbb{C}^{i j k} n_{k}=2(\mathfrak{P}+\mathfrak{R}) \mathfrak{C}^{i j k} n_{k}=0 .
$$

The condition (8.13) is satisfied if and only if the scalar parts of the couplestress distributions coincide at each point of the boundary. It also requires that the arbitrary function $\mathfrak{P}$ of $(8.9)$ and $(8.10)$ be constant on the closed surface. But if $\mathfrak{B}$ is constant on this surface, then grad $\mathfrak{P}$ is parallel to the normal $n$, and the first condition (8.12) will be satisfied automatically. This proves

Theorem 8.2. A necessary and sufficient condition that an energetically equivalent pair of stress and couple-stress distributions be equipollent and have the same stress vector and couple-stress vector at each point of the boundary of a region is that the antisymmetric parts be related by

$$
\begin{aligned}
t^{[i j]} & =t^{*[i]]}+\mathfrak{G}^{i j k} \mathfrak{B}_{, k}{ }^{\prime}, \\
\mathfrak{M} & =\mathfrak{M}^{*}+2 \mathfrak{B},
\end{aligned}
$$

where $\mathfrak{P}$ is an arbitrary scalar field which vanishes on the boundary of the region.

The results summarized in Theorem 8.1 and 8.2 shed some light, we believe, on the indeterminate character of the antisymmetric parts of stress and couplestress in the theory of elasticity presented in the foregoing sections. We are still uncertain as to the physical implications of this indeterminacy, if indeed there be any.

\section{Sound waves in elastic materials with couple-stresses}

The equations of motion of a homogeneous elastic material can be expanded to read as follows:

$$
\begin{aligned}
& \varrho_{0} \ddot{x}_{i}=\frac{\partial^{2} U}{\partial x_{; \alpha}^{i} \partial x_{; \beta}^{i}} x_{; \alpha \beta}^{i}+2 \frac{\partial^{2} U}{\partial x_{; \alpha}^{i} i \partial x_{; \beta \gamma}^{i]}} x_{; \alpha \beta \gamma}^{i}- \\
& -\frac{\partial^{2} U}{\partial x_{; \alpha \beta}^{i} \partial x_{; \gamma \delta}^{i}} x_{; \alpha \beta \gamma \delta}^{j}-\frac{\partial^{8} U}{\partial x_{; \alpha \beta}^{i} \partial x_{; \gamma}^{i} \partial x_{; \sigma}^{\bar{k}}} x_{; \sigma \beta}^{k} x_{; \gamma \alpha}^{j}- \\
& -\frac{\partial^{3} U}{\partial x_{; \alpha \beta}^{i} \partial x_{; \gamma \sigma}^{j} \partial x_{; \varrho}^{k}} x_{; \ell \beta}^{k} x_{; \delta \sigma \alpha}^{j}-\frac{\partial^{3} U}{\partial x_{; \alpha \beta}^{i} \partial x_{; \gamma \delta}^{j} \partial x_{; \alpha \varrho}^{k}} x_{; \gamma \delta \alpha}^{j} x_{; \sigma \rho \beta}^{k} .
\end{aligned}
$$

As is obvious from formulas like (9.1), the theory is complicated, but two interesting features emerge in a general way quite easily. These concern the propagation of plane sound waves. Suppose we have given any solution of the equations (9.1) corresponding to a homogeneous deformation of the material. One such solution is always the undeformed state. Let us represent sound by a "small deformation" superimposed upon the undeformed or initially deformed equilibrium state of the material. In rectangular Cartesian coordinates we put

$$
\hat{x}^{i}(\boldsymbol{X}, t)={ }_{0} \hat{x}^{i}(\boldsymbol{X}, t)+u^{i}(\boldsymbol{X}, t)
$$

where ${ }_{\mathrm{o}} \hat{\boldsymbol{x}}^{i}(\boldsymbol{X}, t)$ satisfies (9.1). When $U$ is independent of second derivatives $x_{; \alpha \beta}$, the equations considered here reduce to those of the usual theory of finite 
elastic deformations. In that theory, the linear equations for small deformations are obtained by substituting (9.2) into (9.1) and casting away all non-linear terms in the gradient of the small displacement $\boldsymbol{u}$. Thus the classical linear theory of elasticity is based upon the assumption that

$$
|\operatorname{grad} \boldsymbol{u}|^{2}=\left(\partial u^{i} / \partial X^{i}\right)\left(\partial u^{i} / \partial X^{j}\right) \ll 1 .
$$

If we follow a similar procedure here, we must also make some assumption about the smallness of the second derivatives, $\partial^{2} u^{2} / \partial X^{i} \partial X^{k}$, if we wish to describe sound by linear equations. To treat this more general case we shall assume that the energy is expressible as accurately as may be desired by a polynomial in the variables $E_{\alpha \beta}$ and $D_{\alpha \beta \lambda}$. Moreover, we shall assume the existence of a material parameter $\mu$ having the physical dimensions of stress, $\left[\mathrm{ML}^{-1} \mathrm{~T}^{\mathbf{2}}\right]$, and a second material parameter $\lambda_{c}$ having the physical dimension of length, $[\mathrm{L}]$, such that, when the energy is expressed in the form

$$
\begin{aligned}
U= & \mu\left[\frac{1}{2} C_{1}^{\mu \nu \alpha \beta} E_{\mu \nu} E_{\alpha \beta}+\frac{\lambda_{c}}{2} C_{2}^{\mu \nu \alpha \beta \lambda} E_{\mu \nu} D_{\alpha \beta \lambda}+\right. \\
& +\frac{\lambda_{c}^{2}}{2} C_{3}^{\mu \nu \alpha \beta \lambda \varrho} D_{\mu \nu \alpha} D_{\beta \lambda \varrho}+\frac{\lambda_{c}^{3}}{6} C_{4}^{\mu \nu \alpha \beta \lambda \varrho \tau \sigma \zeta} D_{\mu \nu \alpha} D_{\beta \lambda \varrho} D_{\tau \sigma \zeta}+\cdots
\end{aligned}
$$

each of the dimensionless material tensors ${ }_{k}, k=1,2,3, \ldots$ has a magnitude of the order of 1 ; i.e.,

$$
|C| \sim 1
$$

where

$$
\left.||_{k}\right|^{2}=C_{k}^{\alpha \beta \cdots}{ }_{k} C_{\alpha \beta \ldots}
$$

Now if for all values of $\boldsymbol{X}$ and $t,|\boldsymbol{E}| \ll 1$ and $\left|\lambda_{c} \boldsymbol{D}\right| \ll 1$, the energy is given approximately by the integral of the first three terms; in (9.4). If it be further assumed that the rotations as well as the strain corresponding to the displacement field $\boldsymbol{u}$ are small throughout the material, then (9.3) holds. The smallness of $\left|\lambda_{c} \boldsymbol{D}\right|$ will imply that $\left|\lambda_{r} \operatorname{grad} \operatorname{grad} \boldsymbol{u}\right|$ is small. Granted all these restrictions on the displacement field $\boldsymbol{u}$ for all $\boldsymbol{X}$ and $t$, it is permissible to linearize the equations (9.1) in $\operatorname{grad} \boldsymbol{u}$ and $\operatorname{grad} \operatorname{grad} \boldsymbol{u}$. The resulting equations may be written in the following form:

$$
\tilde{\varrho} \ddot{u}_{i}=\mu\left[C_{i}^{k} k u_{, k l}^{i}+\lambda_{c} C_{2}^{k l i}{ }^{k l m} u_{, k l m}^{j}-\lambda_{c}^{2} C_{3}^{k l m n} u_{, k l m n}^{i}\right]
$$

where the coefficients $\underset{1}{C}, \underset{2}{C}$, and $\underset{3}{C}$ are constants determined by the form of the energy function and the values of the deformation gradient of the homogeneous solution ${ }_{0} \widehat{\boldsymbol{x}}(\boldsymbol{X})$. The coefficients in (9.7) are given in terms of the energy function by the formulas,

$$
\begin{aligned}
C_{1}^{k l} & =j \frac{\partial^{2} u}{\partial x_{; \alpha}^{i} \partial x_{; \beta}^{j}} x_{; \alpha}^{k} x_{; \beta}^{l}, \\
\lambda_{c} C_{i j}^{k l m} & =2 j \frac{\partial^{2} u}{\partial x_{; \alpha}^{[j} \partial x_{; \beta \lambda}^{i]}} x_{; \alpha}^{k} x_{; \beta}^{l} x_{; \lambda}^{m}, \\
\lambda_{\sigma}^{2} C_{i j}^{k l m n} & =j \frac{\partial^{2} u}{\partial x_{; \alpha \beta}^{i} \partial x_{; \lambda \ell}^{j}}-x_{; \alpha}^{k} x_{; \beta}^{l} x_{; \lambda}^{m} x_{; \varrho}^{n},
\end{aligned}
$$


where all the quantities on the right are evaluated at the solution ${ }_{0} \widehat{\boldsymbol{x}}(\boldsymbol{X})$. The quantity $\tilde{\varrho}$ which appears in (9.7) is the density of mass in the homogeneously deformed state, and the derivatives which appear are with respect to the deformed coordinates $o \hat{x}^{i}$, and $j=\tilde{\varrho} / \varrho_{0}$.

In the way described above, the problem of small deformations is reduced to a study of the system of linear equations with constant coefficients (9.7).

In any material which has a center of symmetry so that the energy is invariant under the transformation

$$
\boldsymbol{X} \rightarrow-\boldsymbol{X}
$$

each of the coefficients $\underset{1}{C_{i j}^{p q r}}=-{\underset{2}{j i}}_{j i}^{p q r}$ vanishes. But in those anisotropic materials which lack a center of symmetry, the terms in the equations of motion involving the coefficients $C_{2 i}^{p q r}$ give rise to an interesting phenomenon which is not embraced by the classical linear theory of elasticity. We shall discuss this phenomenon in a moment, but first we digress to insert some purely kinematical apparatus on plane vector waves which will allow an easy expression of the ideas involved.

A plane vector wave is a vector field of the form

where

$$
\boldsymbol{u}(\boldsymbol{x}, t)=\mathscr{R} \boldsymbol{a} e^{i \varphi}
$$

$$
\varphi=k \boldsymbol{n} \cdot \boldsymbol{x}-\omega t
$$

is its complex phase; $\omega$ is a positive real number called the angular frequency; $\boldsymbol{n}$ is a real unit vector normal to the plane of the wave; and $k$ is the complex wave number. The frequency is given by $f=\omega / 2 \pi$. Let $^{1} k=k^{+}+i k^{-}$, where $k^{+}$and $k^{-}$ are the real and imaginary parts of the wave number. When $k^{+} \neq 0$, the wave is progressive and its speed is defined by $s=|\omega / k|$. The wave length of a progressive wave is defined by $\lambda=\left|1 / k^{\top}\right|$, the direction of propagation by $\overline{\boldsymbol{n}}=\left(\operatorname{sgn} k^{+}\right) \boldsymbol{n}$. The dimensionless number $\eta=k-\mid k=\tan ^{-1} \vartheta$, where $\vartheta$ is the argument of $k$, is called the coefficient of decay if positive and of growth if negative. The complex vector $\boldsymbol{a}=\boldsymbol{a}^{+}+i \boldsymbol{a}^{-}$is the complex amplitude of the wave. We then have

$$
\boldsymbol{u}=e^{-\eta} \frac{\overline{\boldsymbol{n}} \cdot \boldsymbol{x}}{\lambda}\left[\boldsymbol{a}^{+} \cos \left(\frac{\overline{\boldsymbol{n}} \cdot \boldsymbol{x}}{\lambda}-\omega t\right)-\boldsymbol{a}^{-} \sin \left(\frac{\overline{\boldsymbol{n}} \cdot \boldsymbol{x}}{\lambda}-\omega t\right)\right],
$$

where all quantities are real and easy to interpret. The two real vectors $\boldsymbol{a}^{+}$ and $\boldsymbol{a}^{-}$when not collinear determine the plane of oscillation; when collinear, the line of oscillation. When the plane of the wave and the plane of oscillation coincide or when the line of oscillation lies in the plane of the wave, the wave is called transverse. When $\boldsymbol{a}^{+}$and $\boldsymbol{a}^{-}$are collinear, the wave is linearly polarized. A linearly polarized wave whose line of oscillation coincides with the direction of propagation is called a longitudinal wave. When $\boldsymbol{a}^{+}$and $\boldsymbol{a}^{-}$are perpendicular to each other and of equal length, the locus of $\boldsymbol{u}$ at a fixed spatial point $\boldsymbol{x}$ is a circle in the plane of oscillation, and the wave is circularly polarized. A necessary

1 Henceforth in this section we distinguish the real and imaginary parts of a scalar or vector quantity by superscripts + and - . 
and sufficient condition that a wave be circularly polarized is that the complex amplitude satisfy the equation $\boldsymbol{a} \cdot \boldsymbol{a}=0$; a necessary and sufficient condition that a wave be linearly polarized is that $\boldsymbol{a} \times \boldsymbol{a}^{*}=0$, or $\boldsymbol{a}=\boldsymbol{\zeta} \boldsymbol{a}^{*}$, where $\boldsymbol{a}^{*}$ denotes the complex conjugate of $a$. If a wave is neither linearly nor circularly polarized, then it is elliptically polarized, and the locus of $\boldsymbol{u}$ at each $\boldsymbol{x}$ is an ellipse in the plane of oscillation. A wave is right-handed if $\left[\boldsymbol{a}^{+} \boldsymbol{a}^{-} \overline{\boldsymbol{n}}\right]<0$, and left-handed if $\left[\boldsymbol{a}^{+} \boldsymbol{a}^{-} \overline{\boldsymbol{n}}\right]<0$.

We wish now to determine the conditions under which the equations (9.7) admit plane wave solutions and to determine their geometry. If we substitute a plane wave of general character into these equations, we find that it will be a solution if and only if the complex amplitude, the complex wave number, the frequency, and the direction of propagation are related by the equation

$$
Q_{i k}(k, \bar{n}) a^{k}=\bar{\varrho} \omega^{2} a_{i}
$$

where the acoustical tensor ${ }^{1} Q_{i k}(k, \bar{n})$ is given by

with

$$
Q_{\imath k}(k, \overline{\mathbf{n}})=k_{1}^{2} Q_{i k}+i k^{3}{\underset{2}{2}}_{i k}+k^{4}{\underset{3}{i k}}_{i}
$$

$$
\begin{aligned}
& Q_{i j}(\overline{\mathbf{n}})=\mu C_{\mathbf{1}}^{k l} \bar{n}_{k} \bar{n}_{1}, \\
& Q_{2}(\overline{\boldsymbol{n}})=\mu C_{2}^{k j}{ }_{i j}^{k m} \bar{n}_{k} \bar{n}_{1} \bar{n}_{m}\left(\operatorname{sgn} k^{+}\right), \\
& Q_{3}(\overline{\boldsymbol{n}})=\mu{\underset{3}{i j}}^{k l m n} \bar{n}_{k} \bar{n}_{1} \bar{n}_{m} \bar{n}_{n} .
\end{aligned}
$$

According to (9.14) the complex amplitude $a$ must be an eigenvector of the acoustical tensor, and the corresponding eigenvalue must be real and positive since $\tilde{\varrho}>0$ and $\omega^{2}>0$. A sufficient condition that (9.14) have three linearly independent eigenvectors with real and positive eigenvalues is that the acoustical tensor be Hermitian positive definite. From the definition of the acoustical tensor (9.15) to (9.16) and the material parameters $\underset{\mathbf{1}}{\boldsymbol{C}}, \underset{\mathbf{2}}{\boldsymbol{C}}$, and $\underset{3}{\boldsymbol{C}}$ given in (9.8) to (9.10) we see that the acoustical tensor of a material is Hermitian for all real values of the wave number $k$. That it also be positive definite for real values of the wave number places a restriction on the energy function $U$.

The following theorem illuminates the physical significance of the coefficients ${ }_{2}^{k l j}{ }_{1 j}^{k l m}$ and the associated tensor $Q_{2}$, which determines the imaginary part of the acoustical tensor when the wave number is real.

Theorem 9.1. If the acoustical tensor is positive definite for a direction of propagation $\overline{\boldsymbol{n}}$ and a real wave number $k$, then there exist three linearly independent plane progressive waves for that direction and wave length. The frequencies, and hence the speeds, will be different, in general. If the coefficients $\underset{2}{\boldsymbol{Q}}(\overline{\boldsymbol{n}})$ are not all zero, then at least one of these three waves will not be linearly polarized; i.e., at least one wave will be circularly or elliptically polarized.

${ }^{1} Q_{i}(\bar{n})$ is what Truesdell calls the acoustical tensor of an elastic material without couple-stresses. Cf. [14]. 
The first part of the theorem is immediate from known properties of Hermitian matrices. The second and interesting part can be proved as follows. Suppose that all three waves are linearly polarized. Then the three linearly independent complex amplitudes $\underset{r}{a}, \Gamma=1,2,3$, each of which is an eigenvector of the acoustical tensor, are proportional to their complex conjugates $\underset{\Gamma}{a^{*}}$. We then have the two sets of equations

$$
\begin{aligned}
& Q_{i}{\underset{\Gamma}{a}}_{\Gamma}^{i}=\tilde{\varrho} \underset{\Gamma}{\omega^{2}} a_{i}, \\
& Q_{i j}^{*} a_{\Gamma}^{j}=\tilde{\varrho} \underset{\Gamma}{\omega^{2}} a_{i},
\end{aligned}
$$

where the second follows from the first upon taking the complex conjugate and using the supposition that $\boldsymbol{a}$ is proportional to $\boldsymbol{a}^{*}$ for each value of $\Gamma$. But, subtracting corresponding members of the second set from the first, we get

$$
Q_{2}{ }_{i j} a_{\Gamma}^{i}=0, \quad \Gamma=1,2,3,
$$

which implies that $\underset{\mathbf{2}}{Q(\bar{n})}=0$. This proves the theorem.

In view of the foregoing result, we call the coefficients ${\underset{2}{2 j}}_{k j}^{k l m}$, the rotary tensor of an elastic material with couple-stresses. The tensor $Q_{i}(\bar{n})$ will be the acoustical activity tensor for the direction of propagation $\overline{\boldsymbol{n}}$ since its presence gives rise to a phenomenon analogous to optical activity. ${ }^{1}$

According to the classical theory of elasticity, where $\underset{\mathbf{2}}{\boldsymbol{C}}=\underset{\mathbf{3}}{\boldsymbol{C}}=\mathbf{0}$, to every frequency and every direction of propagation there correspond three linearly independent amplitudes $\boldsymbol{a}$ such that $\boldsymbol{a}=\zeta \boldsymbol{a}^{*}$ (linearly polarized plane waves) with real wave numbers $k$ provided only that the acoustical tensor $Q_{1},(\overline{\boldsymbol{n}})$ is positive definite. The speed of each of these waves is independent of the frequency, so that all materials, in the classical theory, are non-dispersive and non-absorptive. The case of materials with couple-stresses considered here is more complicated. The simplest example of waves in an undeformed, isotropic material suffices to illustrate the new phenomena of the more general theory. For these materials we have

$$
{ }_{2}^{C_{i j}^{l m}}=0 \quad \text { (isotropic material symmetry), }
$$

and, choosing $\mu>0$ as the shear modulus for the material, the dimensionless coefficients ${\underset{1}{i j}}_{i j}^{k l}$ have the familiar form

$$
\begin{aligned}
{ }_{1}^{C_{i j}^{k l}} & =\frac{1}{\mu}\left[\lambda \delta_{i}^{k} \delta_{j}^{l}+\mu\left(g_{i} g^{k l}+\delta_{i}^{l} \delta_{j}^{k}\right)\right], \\
& =g_{i j} g^{k l}+\delta_{i}^{l} \delta_{j}^{k}+\frac{2 \sigma}{1-2 \sigma} \delta_{i}^{k} \delta_{j}^{l},
\end{aligned}
$$

where $\lambda$ and $\mu$ are the Lamé constants for the material and $\sigma$ is its Poisson's ratio. Thus the part $Q_{i},(\bar{n})$ of the acoustical tensor for an undeformed isotropic

1 Acoustical activity is mentioned by V. P. Silin [15] who estimates that it might reach an observable level at frequencies of $10^{\circ}$ to $10^{10}(\mathrm{Sec} \text {. })^{-1}$. Acousţical activity can be induced in an elastic dielectric by a strong magnetic field. This will be discussed in a forthcoming paper generalizing the results of [13]. 
material has the form

$$
\frac{Q_{i j}(\overline{\boldsymbol{n}})}{\mu}=g_{i j}+\frac{n_{i} n_{j}}{1-2 \sigma},
$$

which is positive definite if and only if the shear modulus and Poisson's ratio satisfy the inequalities

$$
\mu>0, \quad-\infty \leqq \sigma<\frac{1}{2} \text { or } \quad 1<\sigma \leqq \infty
$$

We shall assume these restrictions on $\mu$ and $\sigma$ to be satisfied. This insures that the wave speeds of the classical theory are real.

Consider next the coefficients $C_{\mathbf{3}}^{k}{ }_{i j}^{k m n}$ and the corresponding part ${\underset{\mathbf{B}}{i j}}_{i}(\overline{\boldsymbol{n}})$ of the acoustical tensor of an undeformed, isotropic material. Here we notice a remarkable difference between the restricted theory of elastic materials with couple-stresses, where the energy function must satisfy the Cosserat relations (7.26), and the more general theory suggested in $\$ 7$ and remarked upon in the footnote of CA 4 in $\$ 5$. In the more general theory without the Cosserat relations, the energy may depend on the second derivatives of the displacement through all of the variables $C_{\alpha \beta, \lambda}$. The part $Q_{\mathbf{3}}(\overline{\boldsymbol{n}})$ of the acoustical tensor under this more general assumption is an arbitrary isotropic symmetric tensor-valued function of the direction of propagation $\overline{\boldsymbol{n}}$ when the material is isotropic and undeformed. Thus we should have

$$
\frac{Q_{i j}}{\mu \lambda_{c}^{2}}=\alpha_{1} g_{i j}+\alpha_{2} n_{i} n_{j},
$$

analogous to the general isotropic form (9.21) of the part $Q_{i j}(\overline{\boldsymbol{n}})$ of the acoustical tensor. The two dimensionless coefficients $\alpha_{1}$ and $\alpha_{2}$ would be independent. But Mindin \& TiERsten have shown that the terms in the energy function (9.4) which are quadratic in the variables $\mathfrak{D}_{\beta}^{\alpha}$ of the Cosserat theory are expressible as a general linear combination of the two scalar invariants

$$
J_{1}=\mathfrak{D}_{\beta}^{\alpha} \mathfrak{D}_{\alpha}^{\beta}, \quad \cdot J_{2}=G_{\alpha \beta} G^{\varrho \sigma} \mathfrak{D}_{{ }^{\alpha}} \mathfrak{D}_{\sigma}^{\beta}
$$

if the material is isotropic. The dimensionless tensor $\underset{\mathbf{B}}{\boldsymbol{C}}$ given by $(9.10)$ then has the following special form:

$$
C_{3}^{k l m^{n} n}=j^{2} \beta \mathbb{C}^{m l}{ }_{i} \mathbb{C}^{k n}{ }_{j}+\alpha_{1} c^{k m}\left(c^{l n} c_{i j}-c^{n}{ }_{i} c_{j}\right) \text {. }
$$

where the tensor $c^{i j}$ is defined by

$$
c^{i j} \equiv G^{\alpha \beta} x_{; \alpha}^{i} x_{; \beta}^{j},
$$

and the dimensionless material constants $\alpha_{1}$ and $\beta$ are independent. But the part $Q_{\mathbf{3}}(\overline{\boldsymbol{n}})$ of the acoustical tensor corresponding to a $\underset{\mathbf{3}}{\boldsymbol{C}}$ of the form $(9.25)$ is

$$
\frac{Q_{i j}}{\mu \lambda_{c}^{2}}=\alpha_{1}\left(g_{i}, \bar{n}_{i} \bar{n}_{j}\right)
$$

when the material is undeformed and $c^{i j}=g^{i j}$. From the point of view of the more general theory which yields (9.23), the Cosserat relations place the restriction

$$
\alpha_{2}=-\alpha_{1}
$$


upon the dimensionless parameters $\alpha_{1}$ and $\alpha_{2}$. Henceforth, let us assume that $Q$ has the general form (9.23). The relation (9.28) may be inserted at any stage to obtain the corresponding results based upon (9.27).

When (9.21) and (9.23) are inserted into the acoustical equation (9.24) it assumes the form

$$
\Omega^{2} a_{i}=\left[\left\{g_{i j}+(1-2 \sigma)^{-1} \bar{n}_{i} \bar{n}_{j}\right\} K^{2}+\left(\alpha_{1} g_{i j}+\alpha_{2} \bar{n}_{i} \bar{n}_{j}\right) K\right] a^{j}
$$

where we have introduced the dimensionless quantities

$$
K=\lambda_{c} k, \quad \Omega^{2}=\frac{\lambda_{c}^{2} \tilde{\varrho} \omega^{2}}{\mu} .
$$

If either $\alpha_{1}$ or $\alpha_{2}$ is not zero, we can always choose the characteristic length $\lambda_{c}$ in such a way that $\left|\alpha_{1}\right|=1$, or $\left|\alpha_{2}\right|=1$. If both $\alpha_{1}$ and $\alpha_{2}$ are zero, (9.29) reverts to the classical form. Jet us assume that $\alpha_{1}$ is not zero and adjust the definition of $\lambda_{c}$ so that $\left|\alpha_{1}\right|=1$. Now the eigenvectors of the acoustical tensor of an undeformed isotropic material are easy to determine by inspection. The direction of propagation is one eigenvector yielding a longitudinal wave, and any vector perpendicular to the direction of propagation is an eigenvector yielding. a transverse wave. For the longitudinal waves we get the dispersion relation

$$
\Omega^{2}=\frac{2(1-\sigma)}{1-2 \sigma} K^{2}+\left(\alpha_{1}+\alpha_{2}\right) K^{4},
$$

and for the transverse waves we get the dispersion relation

where $\alpha_{1}= \pm 1$.

$$
\Omega^{2}=K^{2}+\alpha_{1} K^{4}
$$

By inspection of (9.31) we perceive the following

Theorem 9.2. The longitudinal wave in an undeformed isotropic clastic material with couple-stresses has a speed independent of its frequency if and only if the relation (9.28), which followes from the Cosserat relations (7.26), holds.

This theorem provides a simple criterion for the validity of the Cosserat relations.

The graph of $\Omega$ vs. $K$ is called the dispersion curve. To each assigned value of the non-dimensional frequency $\Omega$ in (9.31) or (9.32), there correspond four non-dimensional wave numbers $K$. Since each equation for $K$ has real coefficients, the roots of these equations for assigned values of the frequency are either two pairs of complex conjugate values of $K$, one pair of complex conjugates . and one pair of reals, or four real roots. The nature of the roots and the shape of the dispersion curves are strongly affected by the sign of the parameter $\alpha_{1}$ in (9.32) or the sign of $\left(\alpha_{1}+\alpha_{2}\right)$ in (9.31). Mindlin \& TIERsten have shown how uniqueness of certain boundary-value problems rests upon the sign of $\alpha_{1}$.

\section{References}

[1] Truesdell, C., \& R. Toupin: The Classical Field Theories, Vol. III/1, Handbuch der Physik, Edited by S. FuÜGGE. Berlin-Göttingen-Heidelberg: Springer 1960.

[2] Cosserat, E. \& F.: Théorie des Corps Déformables. Paris: Hermann 1909.

[3] ErICKSEN, J. L.: Anisotropic fluids. Arch. Rational Mech. Anal. 4, 231-237 (1960). 
[4] ERICKSEN, J. L.: Conservation laws for liquid crystals. Trans. Soc. of Rheology 5, 23-24 (1961).

[5] ERICKsEn, J. L.: Transversely isotropic fluids. Kolloid-Z. 173, 117-122 (1960).

[6] ERICKSEN, J. L.: Theory of anisotropic fluids. Trans. Soc. of Rheology 4, 29-39 (1960).

[7] WEYL, H.: The Classical Groups, Their Invariants and Representations. Princeton University Press 1946.

[8] WhitNey, H.: Geometric Integration Theory. Princeton University Press 1957.

[9] WeyL, H.: The Theory of Groups and Quantum Mechanics. New York: Dover 1932.

[10] Schouten, J. A.: Der Ricci-Kalkül. Berlin: Springer 1924.

[11] Litrlewood, D. E.: The Theory of Group Characters and Matrix Representations of Groups. Oxford: The Clarendon Press 1950.

[12] WADE, T. L.: Tensor algebra and Young's symmetry operators. Amer. J: Math. 63, 645-657 (1941).

[13] Toupin, R. A.: The elastic dielectric. J. Ratl. Mech. and Anal. 5, 849-916 (1956).

[14] Truespell, C.: General and exact theory of waves in finite elastic strain. Arch. Rational Mech. Anal. 8, 263-296 (1961).

[15] Silin, V. P.: Contribution to the theory of absorption of ultrasound in metals. JETP (U.S.S.R.) 38, 977-983 (1960); 11, 703 of the Amer. Phys. Soc. translations.

Mathematical Sciences Division Thomas J. Watson Research Center Yorktown Heights, N.Y. 